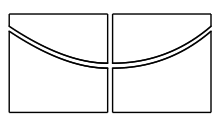

\author{
Universidade de Brasília \\ Instituto de Ciências Exatas \\ Departamento de Estatística
}

Dissertação de Mestrado

Família de distribuições gama tipo III generalizada

por

Adélio Henrique da Silva Neto

Orientador: Prof. Dr. Antônio Eduardo Gomes

Junho de 2016 


\title{
Família de distribuições gama tipo III generalizada
}

\begin{abstract}
Dissertação apresentada ao Departamento de Estatística do Instituto de Ciências Exatas da Universidadede de Brasília como requisito parcial à obtenção do título de Mestre em Estatística.
\end{abstract}

Universidade de Brasília

Brasília, Junho de 2016 
Termo de AprovaÇão

Adélio Henrique da Silva Neto

\section{FAMÍlia DE DISTRIBUiÇÕES GAMA TIPO III GENERALIZADA}

Dissertação apresentada ao Departamento de Estatística do Instituto de Ciências Exatas da Universidadede de Brasília como requisito parcial à obtenção do título de Mestre em Estatística.

Data da defesa: 30 de Junho de 2016

Orientador:

Prof. Dr. Antônio Eduardo Gomes

Departamento de Estatística, UnB

Comissão Examinadora:

Profa. Dra. Juliana Betini Fachini Gomes

Departamento de Estatística, UnB

Prof. Dr. Marcelo Bourguignon Pereira

Departamento de Estatística, UFRN

Brasília, Junho de 2016 
Ficha Catalográfica

\section{DA SILVA NETO, ADÉLIO HENRIQUE}

Família de distribuições gama tipo III generalizada, (UnB - IE, Mestre em Estatística, 2016).

Dissertação de Mestrado - Universidade de Brasília. Departamento de Estatística - Instituto de Ciências Exatas.

1. Família Gama tipo III Generalizada 2. Distribuição Burr III

3. Distribuição Weibull 4. Entropia

É concedida à Universidade de Brasília a permissão para reproduzir cópias desta dissertação de mestrado e para emprestar ou vender tais cópias somente para propósitos acadêmicos e científicos. O autor reserva outros direitos de publicação e nenhuma parte desta dissertação de mestrado pode ser reproduzida sem a autorização por escrito do autor.

Adélio Henrique da Silva Neto 
A Deus, família

e amigos. 


\section{Agradecimentos}

- Agradeço, primeiramente, a Deus por me ter guiado até aqui.

- A minha família pela compreensão, carinho e apoio em todos as circunstâncias da vida.

- Aos amigos que nos ajudam melhor a compreender o sentido da vida.

- E, finalmente, ao meu orientador pela sua dedicação. 


\section{Sumário}

$\begin{array}{ll}\text { Lista de Figuras } & 4\end{array}$

Lista de Tabelas $\quad 5$

$\begin{array}{ll}\text { Resumo } & 6\end{array}$

$\begin{array}{ll}\text { Abstract } & 7\end{array}$

$\begin{array}{lr}\text { Introdução } & 8\end{array}$

1 Uma Nova Família de Distribuições $\quad 11$

1.1 Expansão da função densidade e momentos . . . . . . . . . . . . . . . 12

1.2 Função quantílica e simulação . . . . . . . . . . . . . . . . . . . . 13

1.3 Entropia . . . . . . . . . . . . . . . . . . . . 14

1.4 Estimação . . . . . . . . . . . . . . . . . . . . . 16

2 Distribuição Gama tipo III Burr III Generalizada 18

2.1 Expansão da função densidade e momentos . . . . . . . . . . . . . . 23

2.2 Função quantílica e simulação . . . . . . . . . . . . . . . . . . . . 24

2.3 Aplicação . . . . . . . . . . . . . . . . . . . . 25

3 Distribuição Gama tipo III Weibull Generalizada 28

3.1 Expansão da função densidade e momentos . . . . . . . . . . . . . . . 33

3.2 Função quantílica e simulação . . . . . . . . . . . . . . . . . . . 36

3.3 Aplicação . . . . . . . . . . . . . . . . . . . . . . 36

4 Conclusões e Trabalhos Futuros 39 
4.1 Conclusões . . . . . . . . . . . . . . . . . . . . . . . . . . . . . . . 39

4.2 Trabalhos Futuros . . . . . . . . . . . . . . . . . 40

$\begin{array}{ll}\text { Referências Bibliográficas } & 41\end{array}$

Apêndice $1 \quad 44$ 


\section{Lista de Figuras}

2.1 Gráficos da função densidade GIIIBIIIG para alguns valores dos parâmetros de forma $a$ e $r \ldots \ldots \ldots \ldots \ldots$

2.2 Gráficos da função densidade GIIIBIIIG para alguns valores dos parâmetros

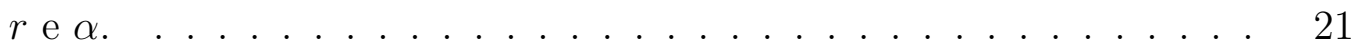

2.3 Gráficos da função densidade GIIIBIIIG para alguns valores do parâmetro de forma $\beta$ em dois cenários diferentes. . . . . . . . . . . . . . 22

2.4 Gráficos da função taxa de falha de GIIIBIIIG para alguns valores dos parâmetros de forma $a, r$, cada um em dois cenários diferentes. . . . . 22

2.5 Gráficos da função taxa de falha de GIIIBIIIG para os parâmetros de forma $\beta$ e $\alpha$, cada um em dois cenários diferentes. . . . . . . . . . . 23

2.6 Assimetria e curtose da distribuição GIIIBIIIG como função de $a$ e $r$. 24

2.7 Assimetria e curtose da distribuição GIIIBIIIG como função de $\alpha$ e $\beta .25$

2.8 Gráfico dos modelos GIIIBIIIG, GIIIBIII e Gama ajustados a um conjunto de dados reais com seu histograma. . . . . . . . . . . . . . 27

3.1 Gráficos da função densidade GIIIWG para alguns valores do parâmetro de forma $r$ em seis cenários diferentes. . . . . . . . . . . . . . . 30

3.2 Gráficos da função densidade GIIIWG para alguns valores dos parâmetros de escala $v$ e de forma $a, k$, cada um em dois cenários diferentes. . . . 3

3.3 Gráficos da função taxa de falha de GIIIWG para alguns valores dos parâmetros de forma $a, r, k$, cada um em dois cenários diferentes. . . 32

3.4 Gráficos da função taxa de falha de GIIIWG para alguns valores do parâmetro de escala $v$ em dois cenários diferentes. . . . . . . . . . 33

3.5 Assimetria e curtose da distribuição GIIIWG como função de $a$ e $r$. 34 
3.6 Assimetria e curtose da distribuição GIIIWG como função de $a$ e $r, k$

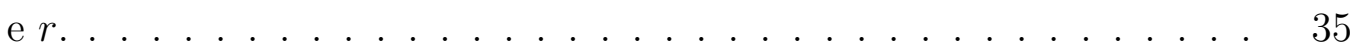

3.7 Gráfico dos modelos GIIIWG, GIIIW, GIIIEG e GIIIE ajustados a um conjunto de dados reais com seu histograma. . . . . . . . . . . . . . 38 


\section{Lista de Tabelas}

2.1 Estimativas de máxima verossimilhança dos parâmetros do modelo GIIIBIIIG e do seus sub-modelos. . . . . . . . . . . . . 26

2.2 Testes de Hipóteses dos modelos GIIIBIIIG versus GIIIBIII e GIIIBIIIG versus Gama com suas respectivas estatísticas w e p-valores. . . .

3.1 Estimativas de máxima verossimilhança dos parâmetros dos modelos GIIIWG, GIIIW, GIIIEG e GIIIE. . . . . . . . . . . . . . 37

3.2 Testes de Hipóteses dos modelos. . . . . . . . . . . . . . . . . . 37 


\section{Resumo}

Na última década, diversos autores têm investigado novas famílias de distribuições de probabilidade com o intuito de obter modelos mais flexíveis para o ajuste a conjunto de dados. Neste trabalho, introduzimos pela primeira vez uma nova família de distribuições referida como a Gama tipo III Generalizada, que é uma generalização da família Gama tipo III (Torabi e Montazeri [6]). Estudamos duas distribuições particulares desta família: a Gama tipo III Burr III Generalizada (GIIIBIIIG) e a Gama tipo III Weibull Generalizada (GIIIWG). Fornecemos, também, uma descrição básica de algumas propriedades matemáticas e computacionais desta nova classe e destas novas distribuições. Para investigar o ajuste das novas distribuições foi analisado para cada modelo um conjunto de dados reais.

Palavras Chave: Familia Gama tipo III Generalizada, distribuição Burr III, distribuição Weibull, Momentos, Entropia, Função quantílica. 


\section{Abstract}

In the last decade, several authors have studied new probability distribution families to obtain more flexible models. In this work, we introduce for the first time the Generalized Gama type III family of distributions wich is a generalization of gama type III family (Torabi and Montazeri [6]). We have studied two specific models of this family: Generalized Gama type III Burr III and Generalized Gama type III Weibull. We provide, also, a description of some of the mathematical and computational properties of this new class of distributions and the studied models. We fitted each model to a data set to investigate their flexibility.

key words: Generalized Gama type III family, Burr III distribution, Weibull distribution, Moments, Entropy, quantile function. 


\section{Introdução}

Um modelo é uma representação simplificada da realidade. Por exemplo, quando utilizamos o Google Maps (ou um Guia de Ruas), na verdade estamos utilizando uma representação mais simples das ruas e avenidas para direcionar o nosso caminho e chegar ao nosso destino. O modelo estatístico funciona de maneira similar, pois através dele podemos representar algum fenômeno ou evento de interesse para nos auxiliar em uma tomada de decisão.

Através das técnicas de modelagem estatística podemos representar processos que possuem incertezas, extrair conhecimento e então optarmos por uma decisão que seja mais favorável aos nossos negócios. Na verdade, em todos os campos de atuação em que existem riscos, incertezas e variações existem aplicações para os modelos estatísticos. Assim como o Google Maps não leva a pessoa ao seu destino, apenas auxilia na sua direção, os modelos estatísticos são ferramentas extremamente valiosas para auxiliar em decisões que envolvam situações de riscos. Dessa forma, a modelagem estatística tem aplicação em diversas áreas como medicina, engenharia, atuária e biologia, entre outras.

Diversas distribuições são comumente empregadas em estudos de modelagem estatística devido às suas propriedades específicas. Contudo, essas distribuições apresentam restrições, como formas limitadas das suas funções de risco, devido ao reduzido número de parâmetros.

A evolução da computação, entretanto, possibilita que distribuições mais complexas sejam ajustadas aos dados. Assim, diversos pesquisadores têm investigado novas maneiras de adicionar parâmetros às distribuições já conhecidas, tornando as distribuições geradas mais flexíveis.

Mudholkar e Srivastava [1], por exemplo, propuseram a distribuição Weibull expo- 
nencializada. As distribuições exponencializadas são construidas da seguinte maneira: seja $Y$ o tempo de falha (tempo até a ocorrência de um evento de interesse) de um componente interpretado como uma variável aleatória com função distribuição $G($.$) .$ Considerando-se, então, um sistema com a componentes em paralelo, em que tal sistema falha se todas as a componentes falharem. Assim, o tempo de falha $X$ do sistema é dado por $X=\max \left\{Y_{1}, Y_{2}, \ldots, Y_{a}\right\}$ e sua função distribuição por

$$
H_{a}(x)=G(x)^{a}
$$

em que $H_{a}(x)$ é chamada de G-exponenializada, por vezes também referida por Ggeneralizada. Sua densidade é representada por

$$
h_{a}(x)=a G(x)^{a-1} g(x) .
$$

A quantidade $a G(x)^{a-1}$ atua como um fator de correção na distribuição original, também, denominada de baseline. É fácil verificar que para $a=1, H_{a}(x)$ é equivalente a baseline $G(x)$, i.e., $H_{1}(x)=G(x)$.

Gupta e Kundu [2] investigaram a distribuição exponencial exponencializada. A distribuição exponencial possui função distribuição e função densidade iguais a

$$
G(x)=1-e^{-x / v} \text { e } g(x)=\frac{1}{v} e^{-x / v}, x>0,
$$

respectivamente, em que $v>0$ é o parâmetro de escala. A distribuição Weibull em que sua função densidade é dada pela Equação (3.2), estudada à frente, com $k=1$, é equivalente a distribuição exponencial. A distribuição e densidade da exponencial exponencializada são

$$
H_{a}(x)=\left(1-e^{-x / v}\right)^{a} \text { e } h_{a}(x)=\frac{a}{v} e^{-x / v}\left(1-e^{-x / v}\right)^{a-1}
$$

respectivamente. Da mesma forma, para $k=1$, a distribuição Weibull (3.2) exponencializada é equivalente a distribuição exponencial exponencializada.

Além da classe G-exponencializada, outro método popular para incluir novos parâmetros em distribuições conhecidas envolve a composição de distribuições. Neste trabalho será considerada apenas a composição de distribuições contínuas com distribuições contínuas. 
Nos próximos capítulos desta dissertação será abordada pela primeira vez uma nova família de distribuições referida como a Gama tipo III Generalizada. No Capítulo 1, fornecemos a idéia de construção desta nova família de distribuições e calculamos algumas de suas propriedades matemáticas e computacionais. No Capítulo 2, estudamos uma distribuição particular desta família, a distribuição Gama tipo III Burr III Generalizada, fornecemos também algumas propriedades matemáticas e computacionais e uma aplicação para um conjunto de dados reais. No Capítulo 3, estudamos outra distribuição particular desta nova classe, a distribuição Gama tipo III Weibull Generalizada, calculamos algumas propriedades matemáticas e computacionais e fornecemos uma aplicação para um conjunto de dados reais. No Capítulo 4, são estabelecidas algumas considerações finais. 


\section{Capítulo 1}

\section{Uma Nova Família de Distribuições}

A literatura estatística sobre distribuições univariadas contínuas é vastíssima. Desenvolvimentos recentes focam em novas técnicas para a construção de distribuições importantes tal como a classe de distribuições beta generalizada (Eugene et al. [3]) que possui dois parâmetros no gerador. Uma distribuição que despertou o interesse de diversos pesquisadores pelo fato de representar uma família paramétrica que possui como casos particulares a distribuição exponencial, Gama, Weibull e Rayleigh é a distribuição Gama Generalizada (GG) que foi introduzida por Stacy [4]. Recentemente, Cordeiro e Castro [5] apresentaram uma nova classe de distribuições a partir da distribuição Kumaraswamy.

Nesse contexto, Torabi e Montazeri [6] propuseram uma nova classe de distribuições a partir da distribuição Gama, que é mencionada em Tahir et al. [7] como a classe de distribuições Gama tipo III. Neste trabalho, propomos a classe ou família de distribuições Gama tipo III Generalizada, que acomoda uma ampla variedade de formas para seus modelos. Com o objetivo de verificar a flexibilidade do parâmetro de generalização $r$, como visto a seguir, e por facilidades matemáticas e computacionais, optamos por tomar o parâmetro de escala do gerador da família Gama tipo III como sendo igual a 1.

Considerando que G denota uma função de distribuição acumulada (fda) de uma variável aleatória contínua, então a distribuição Gama tipo III G Generalizada (GIIIGG) é definida como

$$
F(x)=I_{G(x)}(a, r)=\frac{1}{\Gamma(a)} \int_{0}^{\left(\frac{G(x)}{1-G(x)}\right)^{r}} t^{a-1} e^{-t} d t,
$$


para $a>0$ e $r>0$. O termo generalizada é devido a potência arbitrária $r$. Tem-se, então, que o suporte da $F$ é o mesmo suporte da $G$.

A função densidade de probabilidade (fdp) correspondente à equação (1.1) tem a forma

$$
f(x)=\frac{r}{\Gamma(a)} \frac{g(x) G(x)^{r a-1}}{[1-G(x)]^{r a+1}} e^{-\left(\frac{G(x)}{1-G(x)}\right)^{r}} .
$$

Aqui, $f(x)$ não será simples de ser estudada, a menos que a fda $G(x)$ e a fdp $g(x)=d G(x) / d x$ tenham expressões analíticas bastante simples. Neste trabalho, estudaremos dois modelos específicos desta família: a distribuição Gama tipo III Burr III Generalizada (GIIIBIIIG) e a distribuição Gama tipo III Weibull Generalizada (GIIIWG).

\subsection{Expansão da função densidade e momentos}

As equações (1.1) e (1.2) podem ser calculadas com o uso de recursos computacionais modernos com capacidades analíticas e numéricas. Alternativamente, obtemos expansões para $F(x)$ e $f(x)$ em termos de somas ponderadas infinitas (ou finitas) de fda's e fdp's, respectivamente, da distribuição G exponencializada com fda $G(x)^{r(a+j)+k}$. Utilizando as expansões

$$
e^{z}=\sum_{j=0}^{\infty} \frac{z^{j}}{j !}, \text { para } z \in \mathbb{R}
$$

e

$$
(1-z)^{-b}=\sum_{i=0}^{\infty} \frac{\Gamma(b+i)}{\Gamma(b)} \frac{z^{i}}{i !}, \text { para }|z|<1, b>0,
$$

a equação (1.2) fica

$$
\begin{aligned}
f(x)= & \frac{r}{\Gamma(a)} g(x) \frac{G(x)^{r a-1}}{[1-G(x)]^{r a+1}} e^{-\left[\frac{G(x)}{1-G(x)}\right]^{r}} \\
= & \frac{r}{\Gamma(a)} \sum_{j=0}^{\infty} \sum_{i=0}^{\infty} \frac{(-1)^{j}}{j ! i !} \frac{\Gamma(r(a+j)+i+1)}{\Gamma(r(a+j)+1) i !} \frac{1}{[r(a+j)+i]} \\
& \times[r(a+j)+i] G(x)^{r(a+j)+i-1} g(x) \\
= & \sum_{j=0}^{\infty} \sum_{i=0}^{\infty} \omega_{j, i} \frac{d}{d x} G(x)^{r(a+j)+i}
\end{aligned}
$$

onde

$$
\omega_{j, i}=\frac{(-1)^{j}}{j ! i !} \frac{\Gamma(r(a+j)+i+1)}{\Gamma(r(a+j)+1) i !} \frac{1}{[r(a+j)+i]}
$$


$\mathrm{e}$

$$
\frac{d}{d x} G(x)^{r(a+j)+i}=[r(a+j)+i] G(x)^{r(a+j)+i-1} g(x),
$$

sendo que $\omega_{j, i}$ é uma constante e $\frac{d}{d x} G(x)^{r(a+j)+i}$ é a fdp de uma variável aleatoria com fda $G(x)^{r(a+j)+i}$. Portanto, usando a combinação linear (1.5), tem-se que a fda de GIIIGG é

$$
F(x)=\sum_{j=0}^{\infty} \sum_{i=0}^{\infty} \omega_{j, i} G(x)^{r(a+j)+i}
$$

e que o momento de ordem $r$ da GIIIGG é

$$
E\left(X^{r}\right)=\sum_{j=0}^{\infty} \sum_{i=0}^{\infty} \omega_{j, i} \mu_{j, i}^{(r)}
$$

onde $\mu_{j, i}^{(r)}$ é o r-ésimo momento da variável aleatoria com fda $G(x)^{r(a+j)+i}$.

A assimetria $\gamma_{1}=\mu_{3} / \mu_{2}^{2 / 3}$ e a curtose $\gamma_{2}=\mu_{4} / \mu_{2}^{2}$ podem ser determinadas através de momentos centrais $\mu_{k}$, onde $k$ é um inteiro positivo. Mais adiante serão mostrados alguns gráficos de assimetria e curtose das distribuições GIIIBIIIG e GIIIWG.

\subsection{Função quantílica e simulação}

A função quantílica da família GIIIGG, $Q(u)=F^{-1}(u)$, é fácil de ser calculada a partir da função quantílica da $\operatorname{Gama}(a, 1), Q_{a, 1}(u)$, bastando inverter (1.1). Tem-se que

$$
u=F(x)=P(X \leq x)=P\left(Y \leq\left[\frac{G(x)}{1-G(x)}\right]^{r}\right)=Q_{a, 1}^{-1}\left(\left[\frac{G(x)}{1-G(x)}\right]^{r}\right),
$$

onde $Y$ é a variável aleatoria com distribuição $\operatorname{Gama}(a, 1)$ e $Q_{a, 1}^{-1}($.$) é a função inversa$ da função $Q_{a, 1}($.$) . Assim sendo,$

$$
\frac{G(x)}{1-G(x)}=\left[Q_{a, 1}(u)\right]^{1 / r}
$$

e

$$
x=G^{-1}\left(\frac{\left[Q_{a, 1}(u)\right]^{1 / r}}{1+\left[Q_{a, 1}(u)\right]^{1 / r}}\right) .
$$

Então, a geração de uma variável aleatoria que segue uma distribuição da família GIIIGG é dada por

$$
X=G^{-1}\left(\frac{\left[Q_{a, 1}(U)\right]^{1 / r}}{1+\left[Q_{a, 1}(U)\right]^{1 / r}}\right)
$$

onde $U$ é variável aleatória uniforme no intervalo $[0,1]$. 


\subsection{Entropia}

A entropia de uma variável aleatória $X$ com densidade $f(x)$ é uma medida da variação de incerteza. Um valor grande de entropia indica uma incerteza nos dados. A entropia de Shannon é dada por

$$
\begin{aligned}
E[-\log f(X)]= & -\log r+\log \Gamma(a)-E[\log g(X)]-(r a-1) E[\log G(X)] \\
& +(r a+1) E[\log (1-G(X))]+E\left[\left(\frac{G(X)}{1-G(X)}\right)^{r}\right] \\
= & -\log r+\log \Gamma(a)-E[\log g(X)]-(r a-1) E\left[\log \left(\frac{G(X)}{1-G(X)}\right)\right] \\
& +2 E\{\log [1-G(X)]\}+E\left[\left(\frac{G(X)}{1-G(X)}\right)^{r}\right] .
\end{aligned}
$$

As esperanças da Equação (1.8) podem ser obtidas facilmente. Usando a Equação (1.4) tem-se que

$$
\begin{aligned}
E[\log g(X)] \stackrel{(1.4)}{=} & \frac{r}{\Gamma(a)} \sum_{j, i=0}^{\infty} \frac{(-1)^{j}}{j ! i !} \frac{\Gamma(r(a+j)+i+1)}{\Gamma(r(a+j)+1)} \\
& \times \int_{0}^{\infty}[\log g(x)] G(x)^{r(a+j)+i-1} g(x) d x \\
= & \frac{r}{\Gamma(a)} \sum_{j, i=0}^{\infty} \frac{(-1)^{j}}{j ! i !} \frac{\Gamma(r(a+j)+i+1)}{\Gamma(r(a+j)+1)} \\
& \times \int_{0}^{1}\left[\log g\left(G^{-1}(u)\right)\right] u^{r(a+j)+i-1} d u \\
= & \frac{r}{\Gamma(a)} \sum_{j, i=0}^{\infty} \frac{(-1)^{j}}{j ! i !} \frac{\Gamma(r(a+j)+i+1)}{\Gamma(r(a+j)+1)} I_{j, i},
\end{aligned}
$$

onde $G(x)=u$ e $I_{j, i}=\int_{0}^{1}\left[\log g\left(G^{-1}(u)\right)\right] u^{r(a+j)+i-1} d u$. Pode-se calcular $I_{j, i}$ para algumas fda's $G(x)$.

A esperança da função escore se anula, em particular $E\left(\frac{\partial l}{\partial a}\right)=0$, e então a Equação (1.10) dada na Seção 1.4 implica que

$$
E\left[\log \left(\frac{G(X)}{1-G(X)}\right)\right]=\frac{\psi(a)}{r} .
$$

Usando a expansão em série

$$
\log (1-x)=-\sum_{l=1}^{\infty} \frac{1}{l} x^{l},
$$


para $x<1$, podemos expandir a seguinte esperança como

$$
\begin{aligned}
E\{\log [1-G(x)]\} \stackrel{(1.9)}{=} & -\sum_{l=1}^{\infty} \frac{1}{l} E\left[G(x)^{l}\right] \\
\stackrel{(1.4)}{=} & -\frac{r}{\Gamma(a)} \sum_{l=1}^{\infty} \sum_{j, i=0}^{\infty} \frac{1}{l} \frac{(-1)^{j}}{j ! i !} \frac{\Gamma(r(a+j)+i+1)}{\Gamma(r(a+j)+1)} \\
& \times \int_{0}^{\infty} G(x)^{r(a+j)+i+l-1} g(x) d x \\
= & \frac{r}{\Gamma(a)} \sum_{l=1}^{\infty} \sum_{j, i=0}^{\infty} \frac{(-1)^{j+1}}{j ! i ! l} \frac{\Gamma(r(a+j)+i+1)}{\Gamma(r(a+j)+1)[r(a+j)+i+l]} .
\end{aligned}
$$

Finalmente, usando a expansão em série dada na Equação (1.3) tem-se que

$$
\begin{aligned}
E\left[\left(\frac{G(X)}{1-G(X)}\right)^{r}\right] \stackrel{(1.3)}{=} & E\left[G(X)^{r} \sum_{h=0}^{\infty} \frac{\Gamma(r+h)}{h ! \Gamma(r)} G(X)^{h}\right] \\
= & \sum_{h=0}^{\infty} \frac{\Gamma(r+h)}{h ! \Gamma(r)} E\left[G(X)^{r+h}\right] \\
= & \sum_{h=0}^{\infty} \frac{\Gamma(r+h)}{h ! \Gamma(r)} \sum_{j, i=0}^{\infty} \frac{(-1)^{j}}{j ! i !} \frac{\Gamma(r(a+j)+i+1)}{\Gamma(r(a+j)+1)} \\
& \times \int_{0}^{\infty} G(x)^{r(a+j+1)+i+h-1} g(x) d x \\
= & \frac{1}{\Gamma(r)} \sum_{h, j, i=0}^{\infty} \frac{(-1)^{j}}{j ! i ! h !} \frac{\Gamma(r+h) \Gamma(r(a+j)+i+1)}{\Gamma(r(a+j)+1)[r(a+j+1)+i+h]}
\end{aligned}
$$

Por fim, obtem-se que

$$
\begin{aligned}
E[-\log f(X)]= & -\log r+\log \Gamma(a)-\frac{r}{\Gamma(a)} \sum_{j, i=0}^{\infty} \frac{(-1)^{j}}{j ! i !} \frac{\Gamma(r(a+j)+i+1)}{\Gamma(r(a+j)+1)} I_{j, i} \\
& -(r a-1) \frac{\psi(a)}{r} \\
& +2 \frac{r}{\Gamma(a)} \sum_{l=1}^{\infty} \sum_{j, i=0}^{\infty} \frac{(-1)^{j+1}}{j ! i ! l} \frac{\Gamma(r(a+j)+i+1)}{\Gamma(r(a+j)+1)[r(a+j)+i+l]} \\
& +\frac{1}{\Gamma(r)} \sum_{h, j, i=0}^{\infty} \frac{(-1)^{j}}{j ! i ! h !} \frac{\Gamma(r+h) \Gamma(r(a+j)+i+1)}{\Gamma(r(a+j)+1)[r(a+j+1)+i+h]} .
\end{aligned}
$$




\subsection{Estimação}

Seja $\boldsymbol{\theta}=\left(a, r, \theta_{1}, \ldots, \theta_{k}\right)^{T}$, onde $k$ é um número natural, o vetor paramétrico da distribuição GIIIGG (1.2). Consideramos o método de máxima verossimilhança para estimar $\boldsymbol{\theta}$. A função $\log$-verossimilhança, $l=l(\boldsymbol{\theta})$, para os $k+2$ parâmetros de uma observação $x$ é

$l(\boldsymbol{\theta})=\log r-\log \Gamma(a)+\log g(x)+(r a-1) \log G(x)-(r a+1) \log (1-G(x))-\left[\frac{G(x)}{1-G(x)}\right]^{r}$.

Os componentes do vetor escore unitário $\mathbf{U}=\left(\partial l / \partial a, \partial l / \partial \beta, \partial l / \partial \theta_{1}, \ldots, \partial l / \partial \theta_{k}\right)^{T}$ são

$$
\begin{aligned}
\frac{\partial l}{\partial a}= & -\psi(a)+r \log \left(\frac{G(x)}{1-G(x)}\right) \\
\frac{\partial l}{\partial r}= & \frac{1}{r}+\left\{a-\left[\frac{G(x)}{1-G(x)}\right]^{r}\right\} \log \left(\frac{G(x)}{1-G(x)}\right) \\
\frac{\partial l}{\partial \theta_{j}}= & \frac{1}{g(x)} \frac{\partial g(x)}{\partial \theta_{j}}+\frac{(r a-1)}{G(x)} \frac{\partial G(x)}{\partial \theta_{j}}+\frac{(r a+1)}{1-G(x)} \frac{\partial G(x)}{\partial \theta_{j}} \\
& -\frac{r[G(x)]^{r-1}}{[1-G(x)]^{r+1}} \frac{\partial G(x)}{\partial \theta_{j}}, j=1, \ldots, k .
\end{aligned}
$$

onde $\psi(a)=\frac{\Gamma^{\prime}(a)}{\Gamma(a)}$ é a função digama.

Para uma amostra aleatória $\left(x_{1}, \ldots, x_{n}\right)$ de tamanho $n$ de $X$, a log-verossimilhança total é $l_{n}=l_{n}(\boldsymbol{\theta})=\sum_{i=1}^{n} l^{(i)}$, onde $l^{(i)}$ é a log-verossimilhança para a $i$-ésima observação $(i=1, \ldots, n)$. A função escore total é $\mathbf{U}_{n}=\sum_{i=1}^{n} \mathbf{U}^{(i)}$, onde $\mathbf{U}^{(i)}$ tem a forma anterior dada para $i=1, \ldots, n$. A estimativa de máxima verossimilhança (EMV) $\hat{\boldsymbol{\theta}}$ de $\boldsymbol{\theta}$ é obtida numericamente das equações não-lineares $\mathbf{U}_{n}=\mathbf{0}$.

A estatística do teste da razão de verossimilhança (RV) pode ser usada para comparar a distribuição GIIIGG com alguns de seus submodelos. Considerando a partição $\boldsymbol{\theta}=\left(\boldsymbol{\theta}_{1}^{T}, \boldsymbol{\theta}_{2}^{T}\right)^{T}$, testes de hipóteses do tipo $H_{0}: \boldsymbol{\theta}_{1}=\boldsymbol{\theta}_{1}^{(0)}$ versus $H_{A}: \boldsymbol{\theta}_{1} \neq$ $\boldsymbol{\theta}_{1}^{(0)}$ podem ser realizados usando a estatística RV dada por $w=2\{l(\hat{\boldsymbol{\theta}})-l(\tilde{\boldsymbol{\theta}})\}$, onde $\hat{\boldsymbol{\theta}}$ e $\tilde{\boldsymbol{\theta}}$ são os EMV's de $\boldsymbol{\theta}$ sob $H_{A}$ e $H_{0}$, respectivamente. Sob a hipótese nula, $w \stackrel{d}{\rightarrow} \chi_{q}^{2}$, onde q é a dimensão do vetor $\boldsymbol{\theta}_{1}$ de interesse. O teste RV rejeita $H_{0}$ se $w>\xi_{\gamma}$, onde $\xi_{\gamma}$ denota o quantil superior de ordem $100 \gamma \%$ da distribuição $\chi_{q}^{2}$. Por exemplo, podemos verificar se o ajuste usando a distribuição GIIIBIIIG é estatisticamente "superior" para um ajuste usando a distribuição GIIIBIII (para um dado conjunto de dados) testando $H_{0}: r=1$ versus $H_{A}: r \neq 1$.

As derivadas parciais de distribuição Burr III (2.2) são dadas por: 


$$
\begin{aligned}
\frac{\partial G}{\partial \alpha}= & {\left[1+\left(\frac{x}{s}\right)^{-\alpha}\right]^{-(\beta+1)} \beta\left(\frac{x}{s}\right)^{-\alpha} \log \left(\frac{x}{s}\right), } \\
\frac{\partial G}{\partial \beta}= & -\left[1+\left(\frac{x}{s}\right)^{-\alpha}\right]^{-\beta} \log \left[1+\left(\frac{x}{s}\right)^{-\alpha}\right], \\
\frac{\partial G}{\partial s}= & -\frac{\beta \alpha}{s}\left[1+\left(\frac{x}{s}\right)^{-\alpha}\right]^{-(\beta+1)}\left(\frac{x}{s}\right)^{-\alpha}, \\
\frac{\partial g}{\partial \alpha}= & \frac{\beta}{x}\left[1+\left(\frac{x}{s}\right)^{-\alpha}\right]^{-(\beta+1)}\left(\frac{x}{s}\right)^{-\alpha}-\frac{\beta \alpha(-\beta-1)}{x}\left[1+\left(\frac{x}{s}\right)^{-\alpha}\right]^{-(\beta+2)} \\
& \times\left(\frac{x}{s}\right)^{-2 \alpha} \log \left(\frac{x}{s}\right)-\frac{\beta \alpha}{x}\left[1+\left(\frac{x}{s}\right)^{-\alpha}\right]^{-(\beta+1)} \log \left(\frac{x}{s}\right)\left(\frac{x}{s}\right)^{-\alpha}, \\
\frac{\partial g}{\partial \beta}= & \frac{\alpha}{x}\left[1+\left(\frac{x}{s}\right)^{-\alpha}\right]^{-(\beta+1)}\left(\frac{x}{s}\right)^{-\alpha}-\frac{\beta \alpha}{x}\left[1+\left(\frac{x}{s}\right)^{-\alpha}\right]^{-(\beta+1)} \log \left[1+\left(\frac{x}{s}\right)^{-\alpha}\right]\left(\frac{x}{s}\right)^{-\alpha} \\
\frac{\partial g}{\partial s}= & \frac{\beta \alpha^{2}(-\beta-1)}{s x}\left[1+\left(\frac{x}{s}\right)^{-\alpha}\right]^{-(\beta+2)}\left(\frac{x}{s}\right)^{-2 \alpha}+\frac{\beta \alpha^{2}}{s x}\left[1+\left(\frac{x}{s}\right)^{-\alpha}\right]^{-(\beta+1)}\left(\frac{x}{s}\right)^{-\alpha} .
\end{aligned}
$$

As derivadas parciais para a distribuição Weibull (3.2) são:

$$
\begin{aligned}
\frac{\partial G}{\partial k} & =\left(\frac{x}{v}\right)^{k} \ln \left(\frac{x}{v}\right) \mathrm{e}^{-\left(\frac{x}{v}\right)^{k}}, \\
\frac{\partial G}{\partial v} & =-\frac{k}{v}\left(\frac{x}{v}\right)^{k} \mathrm{e}^{-\left(\frac{x}{v}\right)^{k}}, \\
\frac{\partial g}{\partial k} & =\frac{x^{k-1}}{v^{k}} \mathrm{e}^{-\left(\frac{x}{v}\right)^{k}}\left[1+k \ln \left(\frac{x}{v}\right)-k\left(\frac{x}{v}\right)^{k} \ln \left(\frac{x}{v}\right)\right], \\
\frac{\partial g}{\partial v} & =\frac{k^{2} x^{k-1}}{v^{k+1}} \mathrm{e}^{-\left(\frac{x}{v}\right)^{k}}\left[\left(\frac{x}{v}\right)^{k}-1\right] .
\end{aligned}
$$




\section{Capítulo 2}

\section{Distribuição Gama tipo III Burr III Generalizada}

Burr [8] introduziu um sistema de distribuições que contém a distribuição Burr XII (BXII). Se a variável aleatória $Y$ tem distribuição BXII, então $X=Y^{-1}$ tem a distribuição Burr III (BIII), que possui função de distribuição acumulada definida por

$$
G_{\alpha, \beta, s}(x)=\left[1+(x / s)^{-\alpha}\right]^{-\beta}=\left[\frac{(x / s)^{\alpha}}{1+(x / s)^{\alpha}}\right]^{\beta}, x>0,
$$

onde $\alpha>0$ e $\beta>0$ são parâmetros de forma e $s>0$ é um parâmetro de escala. A função densidade de probabilidade correspondente a (2.1) é dada por

$$
g_{\alpha, \beta, s}(x)=\frac{\alpha \beta}{s(x / s)^{\alpha+1}}\left[\frac{(x / s)^{\alpha}}{1+(x / s)^{\alpha}}\right]^{\beta+1}, x>0 .
$$

A distribuição BIII tem sido usada em vários campos da ciência. Ela foi inicialmente denominada distribuição Dagum [9, 10] em estudos de distribuição de renda e salário como de distribuição de riquezas e suas características amplamente analizadas. Para um excelente estudo sobre a gênese e aplicações empíricas ver Kleiber e Kotz [11] e Kleiber [12]. Na literatura atuarial é conhecida como distribuição Burr inversa [13] e como distribuição kappa na literatura meteorológica $[14,15]$. Tem sido empregada em finanças, estudos do meio ambiente, análise de sobrevivência e teoria da confiabilidade $[16,17,18,19,20,21,22]$. Além disso, Shao et al. [23] propôs uma distribuição BIII estendida em análise de baixa frequência onde sua cauda inferior é de principal interesse. Uma extensão bivariada da distribuição BIII foi definida por Rodriguez 
[24].

A distribuição Gama tipo III Burr III Generalizada (GIIIBIIIG) com cinco parâmetros, é definida tomando $G(x)$ como sendo a fda de uma variável aleatória Burr III (2.1). Daí obtemos

$$
F(x)=I_{\left[1+(x / s)^{-\alpha}\right]^{-\beta}}(a, r)=\frac{1}{\Gamma(a)} \int_{0}^{\left\{\left[1+(x / s)^{-\alpha}\right]^{\beta}-1\right\}^{-r}} t^{a-1} e^{-t} d t, x>0,
$$

onde $a>0, r>0, \alpha>0, \beta>0$ e $s>0$. A função densidade e a função taxa de falha correspondentes à equação (2.3) são dadas por:

$$
f(x)=\frac{r}{\Gamma(a)} \frac{\alpha \beta}{s(x / s)^{\alpha+1}} \frac{\left[1+(x / s)^{-\alpha}\right]^{-\beta r a-1}}{\left\{1-\left[1+(x / s)^{-\alpha}\right]^{-\beta}\right\}^{r a+1}} e^{-\left\{\left[1+(x / s)^{-\alpha}\right]^{\beta}-1\right\}^{-r}}, x>0
$$

e

$$
\begin{aligned}
h(x)= & \frac{r}{\Gamma(a)} \frac{\alpha \beta}{s(x / s)^{\alpha+1}} \frac{\left[1+(x / s)^{-\alpha}\right]^{-\beta r a-1} e^{-\left\{\left[1+(x / s)^{-\alpha}\right]^{\beta}-1\right\}^{-r}}}{\left\{1-\left[1+(x / s)^{-\alpha}\right]^{-\beta}\right\}^{r a+1}\left(1-I_{\left[1+(x / s)^{-\alpha}\right]^{-\beta}}(a, r)\right)}, \\
& x>0
\end{aligned}
$$

respectivamente. A função densidade da GIIIBIIIG (2.4) inclui dois submodelos importantes: a distribuição Gama tipo III Burr III surge tomando $r=1$ e a distribuição $\operatorname{Gama}(a, 1)$ que surge quando $r=\alpha=\beta=s=1$. Se $X$ é uma variável aleatória com densidade (2.4), então escrevemos $X \sim \operatorname{GIIIBIIIG}(a, r, \alpha, \beta, s)$.

Gráficos da função densidade (2.4) para valores selecionados de $a, r, \alpha, \beta, s$ são dados nas Figuras 2.1, 2.2 e 2.3. Foram escolhidos oito cenários para variar o parâmetro $r$ e dois para os outros parâmetros. O parâmetro $r$ extra de forma da GIIIBIIIG fornece maior flexibilidade na forma da distribuição gerada e, com isso, é mais útil para a modelagem de dados positivos.

As Figuras 2.4 e 2.5 mostram as funções de taxa de falha da GIIIBIIIG (2.5) que podem ter formato de banheira, monotonicamente decrescente, monotonicamente crescente ou unimodal, dependendo dos valores dos parâmetros. 


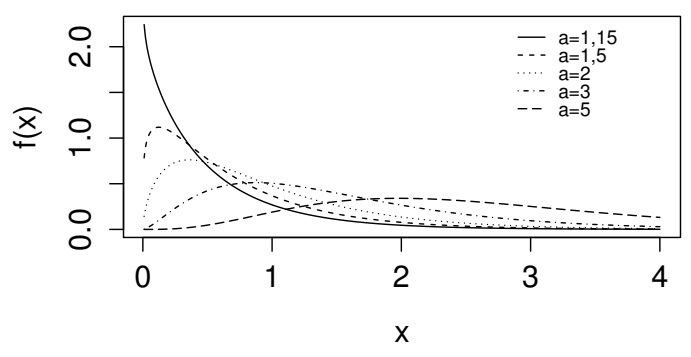

(a) GIIIBIIIG(a,r=1, $\alpha=0,9, \beta=0,9, \mathrm{~s}=0,5)$

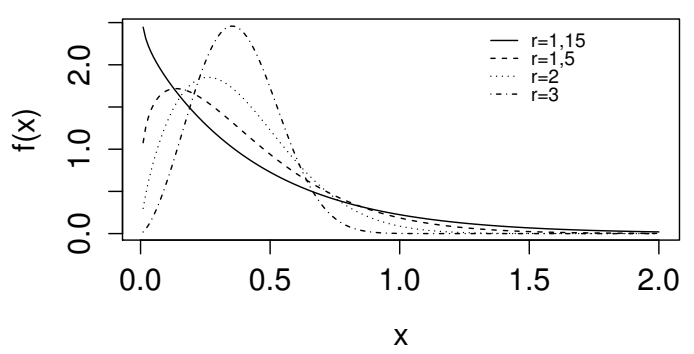

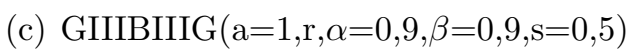

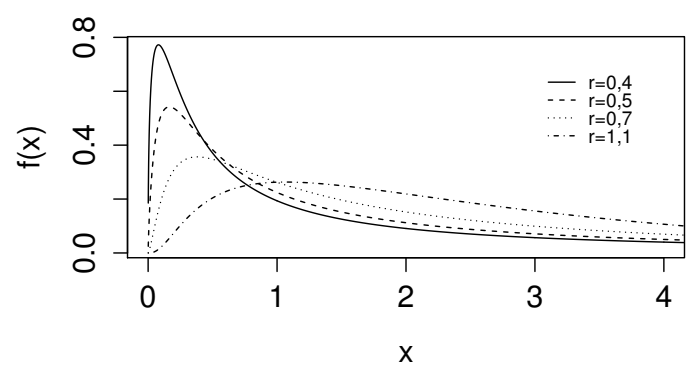

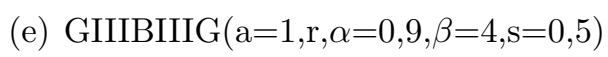

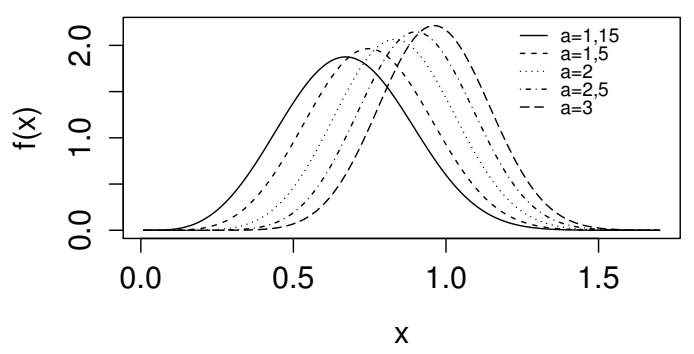

(b) GIIIBIIIG(a,r $=2, \alpha=1,5, \beta=1,5, \mathrm{~s}=0,5)$

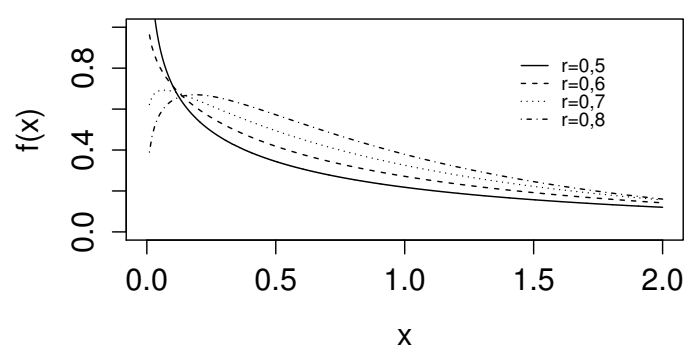

(d) GIIIBIIIG(a $=2, \mathrm{r}, \alpha=0,9, \beta=0,9, \mathrm{~s}=0,5)$

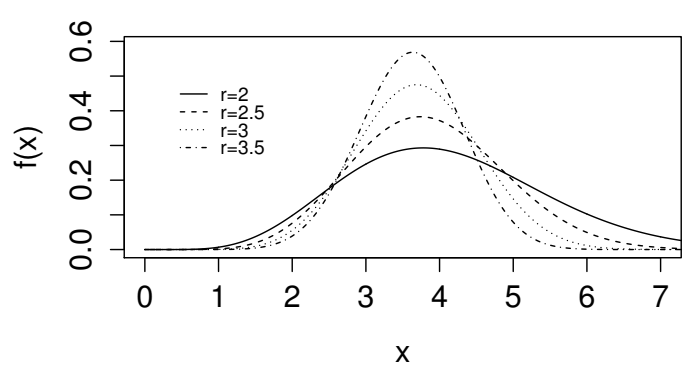

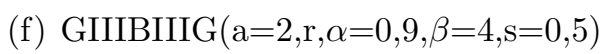

Figura 2.1: Gráficos da função densidade GIIIBIIIG para alguns valores dos parâmetros de forma $a$ e $r$. 


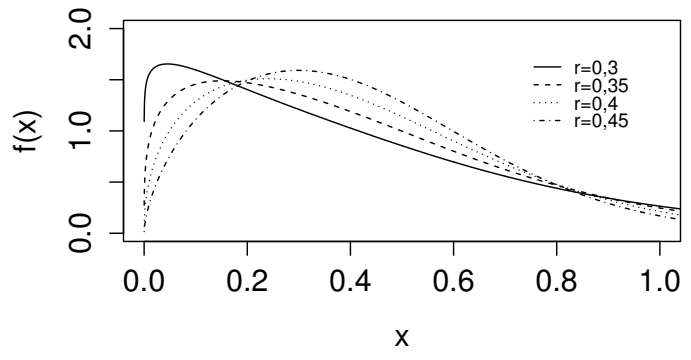

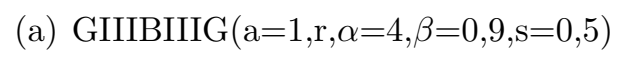

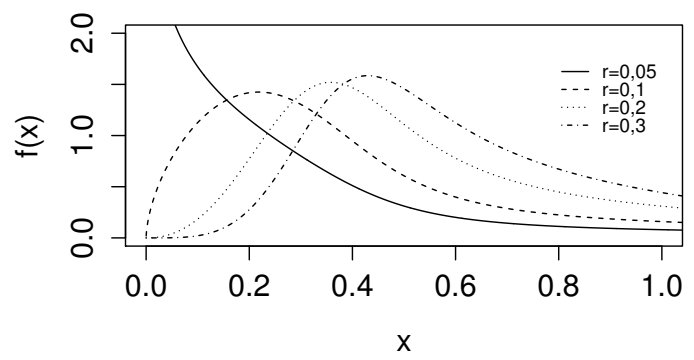

(c) GIIIBIIIG( $\mathrm{a}=1, \mathrm{r}, \alpha=4, \beta=4, \mathrm{~s}=0,5)$

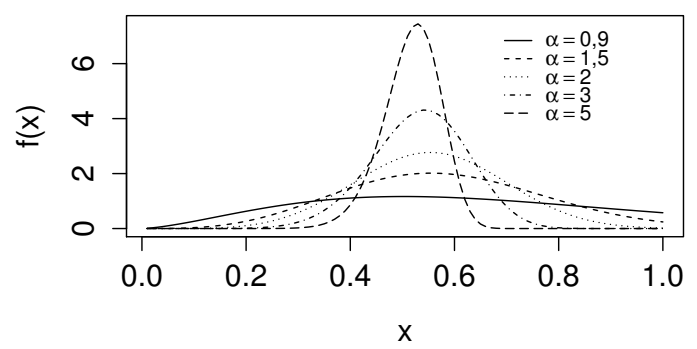

(e) GIIIBIIIG( $\mathrm{a}=2, \mathrm{r}=1,5, \alpha, \beta=0,9, \mathrm{~s}=0,5)$

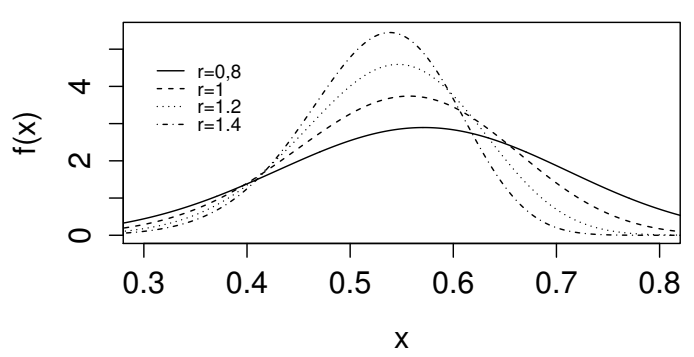

(b) GIIIBIIIG(a $=2, \mathrm{r}, \alpha=4, \beta=0,9, \mathrm{~s}=0,5)$

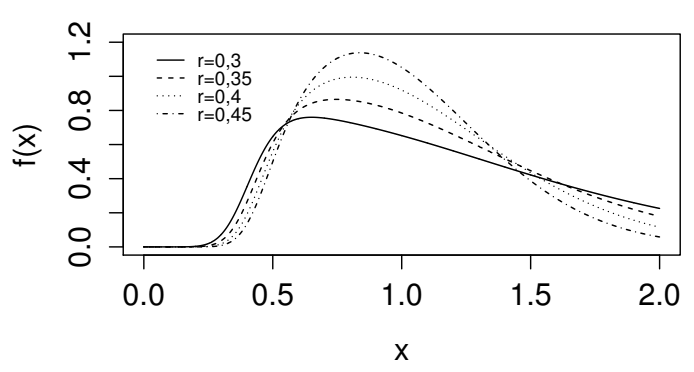

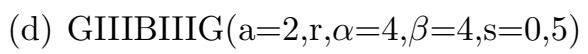

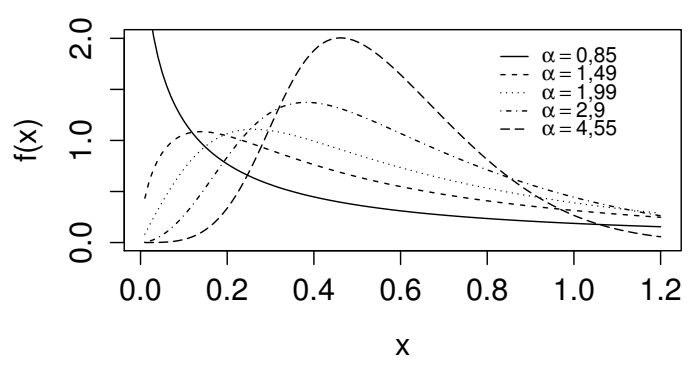

(f) $\operatorname{GIIIBIIIG(}(\mathrm{a}=1, \mathrm{r}=0,5, \alpha, \beta=2, \mathrm{~s}=0,5)$

Figura 2.2: Gráficos da função densidade GIIIBIIIG para alguns valores dos parâmetros $r$ e $\alpha$. 


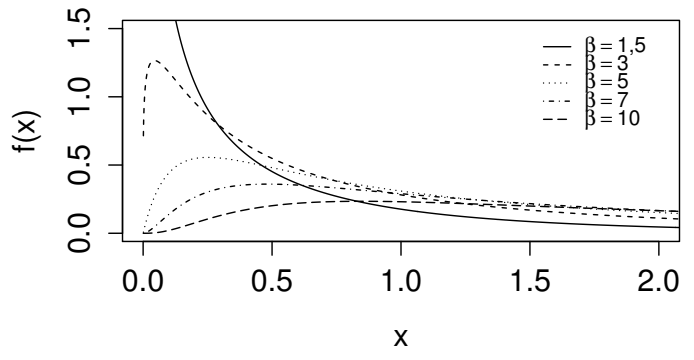

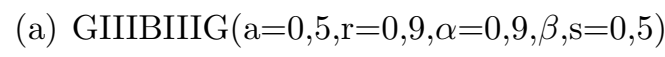

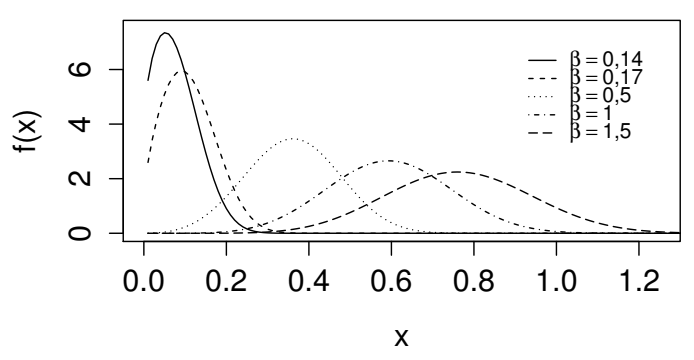

(b) GIIIBIIIG( $\mathrm{a}=2, \mathrm{r}=1,5, \alpha=2, \beta, \mathrm{s}=0,5)$

Figura 2.3: Gráficos da função densidade GIIIBIIIG para alguns valores do parâmetro de forma $\beta$ em dois cenários diferentes.

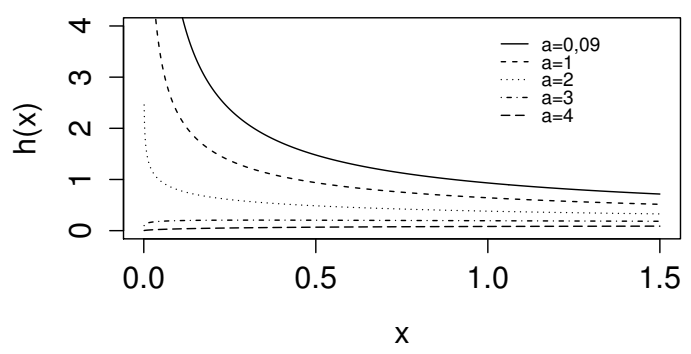

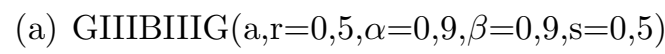

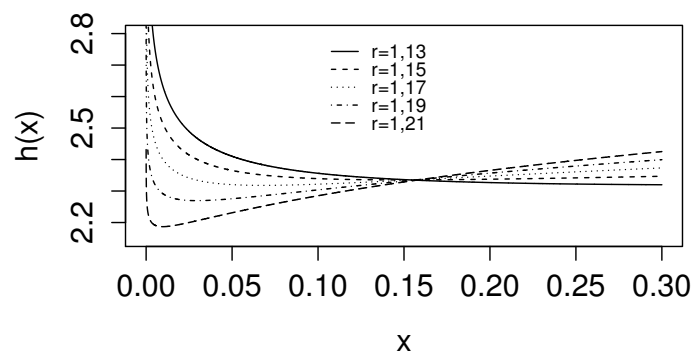

(c) GIIIBIIIG( $(\mathrm{a}=1, \mathrm{r}, \alpha=0,9, \beta=0,9, \mathrm{~s}=0,5)$

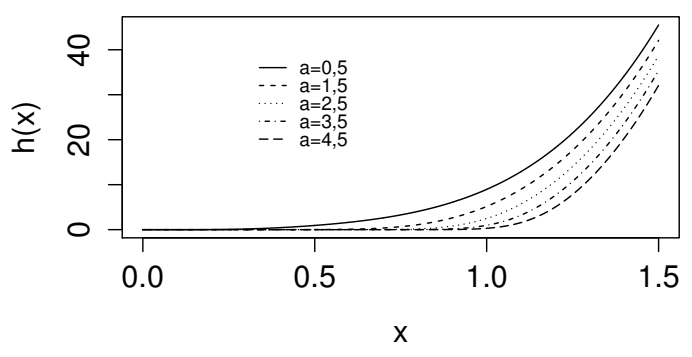

(b) GIIIBIIIG(a,r $=2,5, \alpha=2, \beta=3, \mathrm{~s}=0,5)$

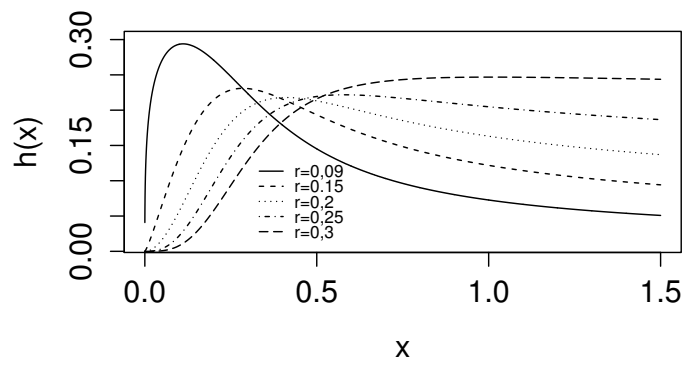

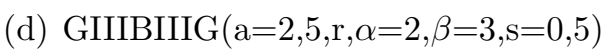

Figura 2.4: Gráficos da função taxa de falha de GIIIBIIIG para alguns valores dos parâmetros de forma $a, r$, cada um em dois cenários diferentes. 


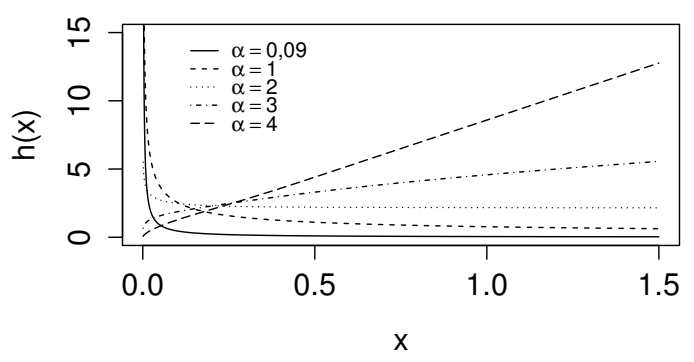

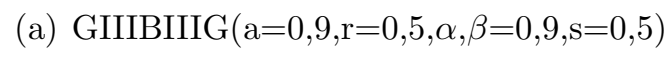

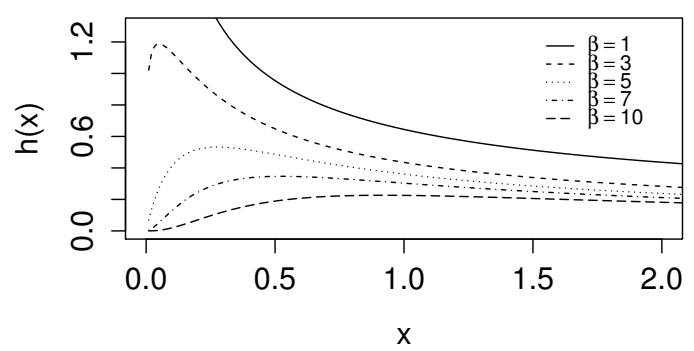

(c) GIIIBIIIG( $(\mathrm{a}=0,9, \mathrm{r}=0,5, \alpha=0,9, \beta, \mathrm{s}=0,5)$

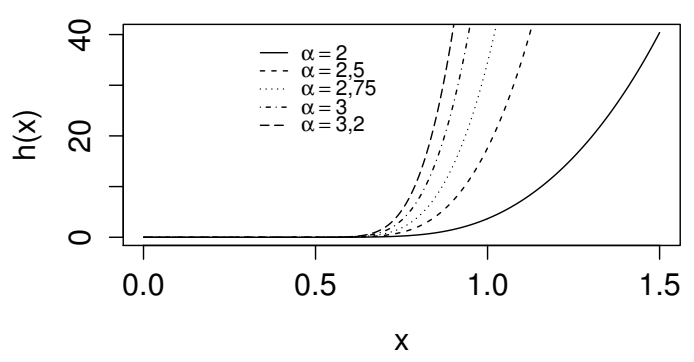

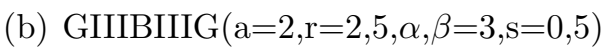

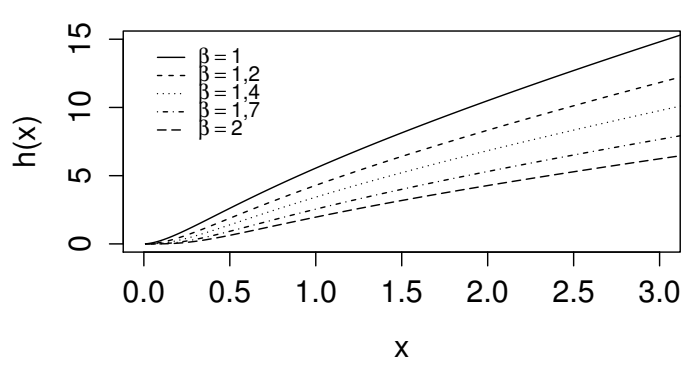

(d) $\operatorname{GIIIBIIIG(}(\mathrm{a}=1,5, \mathrm{r}=1,2, \alpha=1,5, \beta, \mathrm{s}=0,5)$

Figura 2.5: Gráficos da função taxa de falha de GIIIBIIIG para os parâmetros de forma $\beta$ e $\alpha$, cada um em dois cenários diferentes.

\subsection{Expansão da função densidade e momentos}

Quando G é a fda da variável aleatoria Burr III (2.1), com parâmetros $\alpha, \beta, s$, tem-se que

$$
G_{\alpha, \beta, s}(x)^{r(a+j)+i}=\left[1+(x / s)^{-\alpha}\right]^{-\beta[r(a+j)+i]} .
$$

Assim sendo, $\mu_{j, i}^{(r)}$ é obtida facilmente, pois $G_{\alpha, \beta, s}(x)^{r(a+j)+i}$ é a fda da distribuição Burr III com parâmetros $\alpha, \beta[r(a+j)+i], s$. Tem-se que o $r$-ésimo momento da Burr III, com parâmetros $\alpha, \beta$, s, é $\mu_{r}^{\prime}=s^{r} \Gamma(\beta+r / \alpha) \Gamma(1+r / \alpha) / \Gamma(\beta)$. Por conseguinte, o momento de ordem $r$ da GIIIGG é

$$
E\left(X^{r}\right)=\sum_{j=0}^{\infty} \sum_{i=0}^{\infty} \omega_{j, i} s^{r} \frac{\Gamma(\beta[r(a+j)+i]+r / \alpha) \Gamma(1+r / \alpha)}{\Gamma(\beta[r(a+j)+i])} .
$$


Gráficos de assimetria $\gamma_{1}$ e curtose $\gamma_{2}$ para valores selecionados de $a$ como função de $r$, para algumas escolhas de $r$ como função de $a$, para algumas escolhas de $\beta$ como função de $\alpha$, e para algumas escolhas de $\alpha$ como função de $\beta$, são mostradas nas Figuras 2.6 e 2.7 .

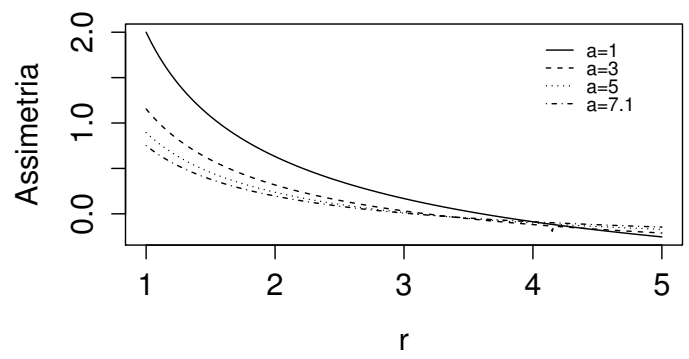

(a) GIIIBIIIG(a,r, $\alpha=1, \beta=1, \mathrm{~s}=1)$

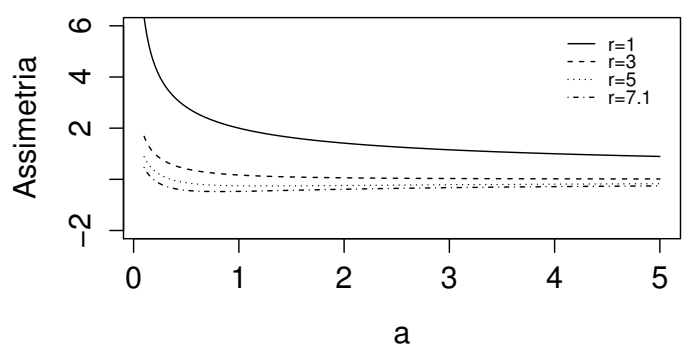

(c) GIIIBIIIG(a,r, $\alpha=1, \beta=1, \mathrm{~s}=1)$

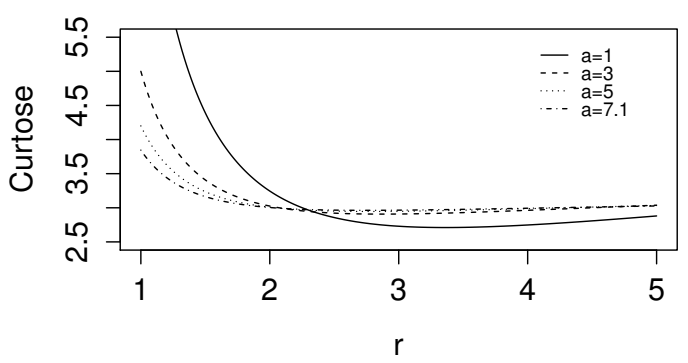

(b) GIIIBIIIG(a,r, $\alpha=1, \beta=1, \mathrm{~s}=1)$

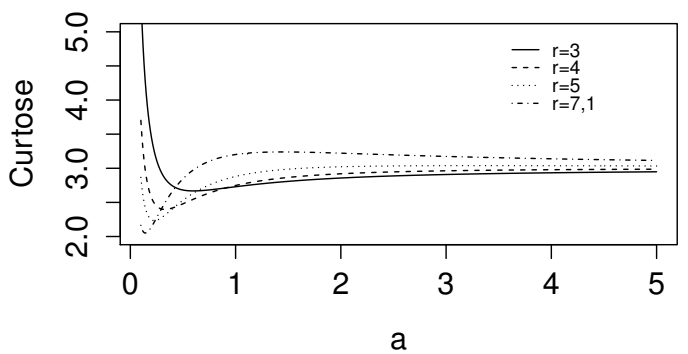

(d) GIIIBIIIG(a,r, $\alpha=1, \beta=1, \mathrm{~s}=1)$

Figura 2.6: Assimetria e curtose da distribuição GIIIBIIIG como função de $a$ e $r$.

\subsection{Função quantílica e simulação}

Seja G a fda da distribuição Burr III (2.1), sua inversa é dada por

$$
G_{\alpha, \beta, s}^{-1}(y)=s\left(y^{-1 / \beta}-1\right)^{-1 / \alpha}
$$

Com isso, da equação (1.7), tem-se que

$$
x=s\left[\left(\frac{\left[Q_{a, 1}(u)\right]^{1 / r}}{1+\left[Q_{a, 1}(u)\right]^{1 / r}}\right)^{-1 / \beta}-1\right]^{-1 / \alpha} .
$$


Por conseguinte, pode-se gerar a variável aleatória GIIIBIIIG pela Equação (2.6), onde $u$ é gerada da distribuição Uniforme $(0,1)$ e $Q_{a, 1}(u)$ está disponível na maioria dos pacotes dos softwares estatísticos.

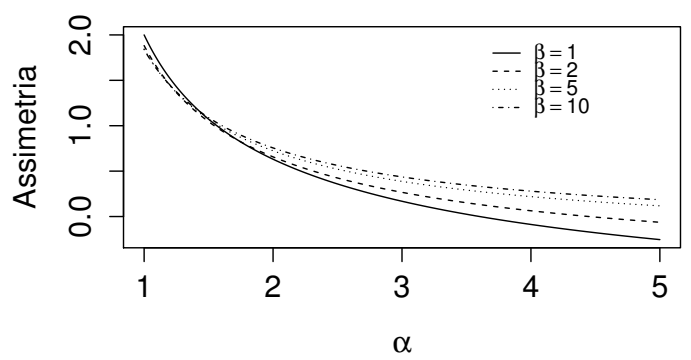

(a) GIIIBIIIG( $(\mathrm{a}=1, \mathrm{r}=1, \alpha, \beta, \mathrm{s}=1)$

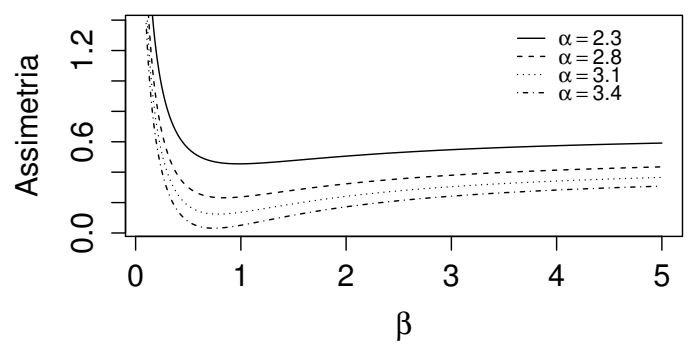

(c) GIIIBIIIG( $(\mathrm{a}=1, \mathrm{r}=1, \alpha, \beta, \mathrm{s}=1)$

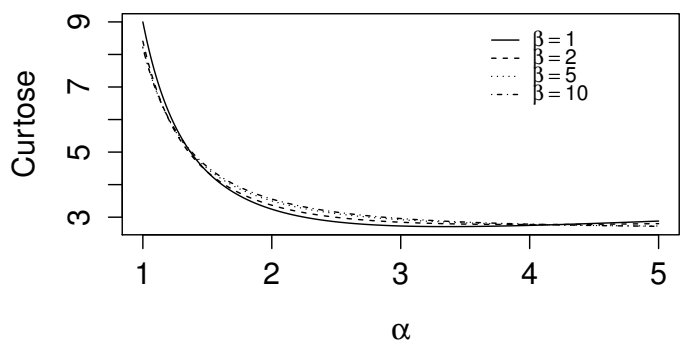

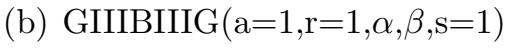

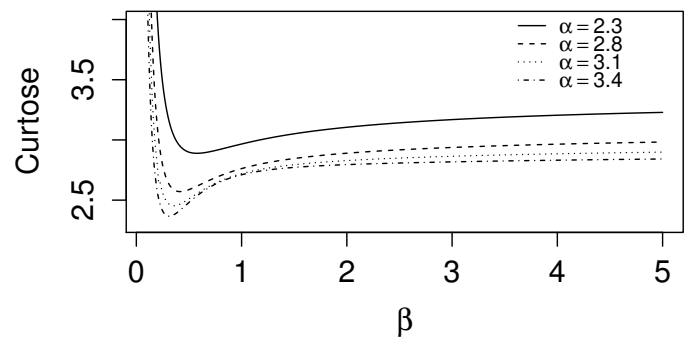

(d) GIIIBIIIG( $(\mathrm{a}=1, \mathrm{r}=1, \alpha, \beta, \mathrm{s}=1)$

Figura 2.7: Assimetria e curtose da distribuição GIIIBIIIG como função de $\alpha$ e $\beta$.

\subsection{Aplicação}

Nesta seção fornecemos uma aplicação usando um conjunto de dados reais para demonstrar a flexibilidade e a potencialidade do modelo GIIIBIIIG.

Para o modelo GIIIBIIIG dado pela equação (2.4) trabalhamos com os seguintes dados que representam o tempo de sobrevivência (em dias) de 72 porquinhos da Índia infectados com "virulent tubercle bacilli", observado e reportado por Bjerkedal [25]. Os dados são como seguem:

$\begin{array}{lllllllllllllllllllllllll}0,1 & 0,33 & 0,44 & 0,56 & 0,59 & 0,72 & 0,74 & 0,77 & 0,92 & 0,93 & 0,96 & 1 & 1 & 1,02 & 1,05 & 1,07 & 1,07 & 1,08\end{array}$ $\begin{array}{lllllllllllllllllll}1,08 & 1,08 & 1,09 & 1,12 & 1,13 & 1,15 & 1,16 & 1,2 & 1,21 & 1,22 & 1,22 & 1,24 & 1,3 & 1,34 & 1,36 & 1,39 & 1,44 & 1,46\end{array}$ 
$\begin{array}{lllllllllllllllll}1,53 & 1,59 & 1,6 & 1,63 & 1,63 & 1,68 & 1,71 & 1,72 & 1,76 & 1,83 & 1,95 & 1,96 & 1,97 & 2,02 & 2,16\end{array}$

2,3 2,31 2,4 2,45 2,51 2,53 2,54 2,54 2,78 2,93 3,27 3,42 3,47 3,61 4,02 4,32 4,58 5,55.

Ajustamos as distribuições GIIIBIIIG, GIIIBIII e Gama aos dados pelo método de máxima verossimilhança. Os EMV's dos parâmetros, com erros padrões (EP), medidos para os modelos são mostrados na Tabela 2.1. Os testes de hipóteses são dados na Tabela 2.2.

A estatística RV para testar as hipóteses $H_{0}$ : GIIIBIII $\times H_{A}$ : GIIIBIIIG e $H_{0}$ : $\mathrm{G} \times H_{A}$ : GIIIBIIIG são 2,908 (p-valor=0,088) e 11,897 (p-valor=0.018), respectivamente. Consequentemente, rejeitamos a hipótese nula em ambos os casos em favor da distribuição GIIIBIIIG a um nível de significância $\alpha=0,10$. Gráficos das densidades ajustadas das distribuições GIIIBIIIG, GIIIBIII e Gama são dados na Figura 2.8. O gráfico indica que a distribuição GIIIBIIIG fornece um melhor ajuste para estes dados do que os sub-modelos GIIIBIII e Gama. Comparada com seus sub-modelos, a distribuição GIIIBIIIG obteve um melhor ajuste em torno da moda e no decaimento das caudas. As implementações, usando o programa R, das estimativas estão no Apêndice.

\begin{tabular}{cccccc}
\hline Modelo & $a$ & $r$ & $\alpha$ & $\beta$ & $s$ \\
\hline GIIIBIIIG & 0,772 & 0,262 & 5,743 & 2,224 & 1,787 \\
& $(0,363)$ & $(0,319)$ & $(3,794)$ & $(2,315)$ & $(0,523)$ \\
GIIIBIII & 4,895 & 1 & 0,863 & 0,509 & 0,662 \\
& $(1,663)$ & $(-)$ & $(0,661)$ & $(2,344)$ & $(0,902)$ \\
Gama & 1,964 & 1 & 1 & 1 & 1 \\
& $(0,136)$ & $(-)$ & $(-)$ & $(-)$ & $(-)$ \\
\hline
\end{tabular}

Tabela 2.1: Estimativas de máxima verossimilhança dos parâmetros do modelo GIIIBIIIG e do seus sub-modelos. 


\begin{tabular}{cccc}
\hline Modelo & Hipóteses & Estatística $\mathrm{w}$ & p-valor \\
\hline GIIIBIIIG x GIIIBIII & $H_{0}: r=1$ & 2.908 & 0.088 \\
GIIIBIIIG x Gama & $H_{0}: r=\alpha=\beta=s=1$ & 11.897 & 0.018 \\
\hline
\end{tabular}

Tabela 2.2: Testes de Hipóteses dos modelos GIIIBIIIG versus GIIIBIII e GIIIBIIIG versus Gama com suas respectivas estatísticas w e p-valores.

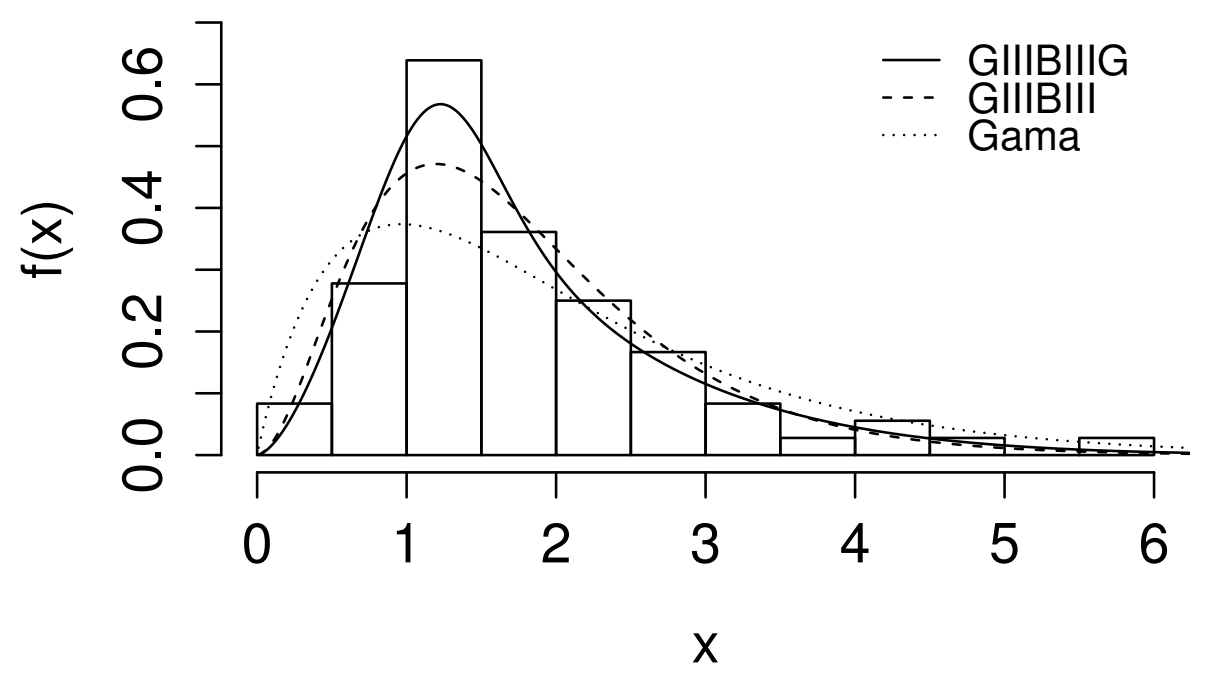

Figura 2.8: Gráfico dos modelos GIIIBIIIG, GIIIBIII e Gama ajustados a um conjunto de dados reais com seu histograma. 


\section{Capítulo 3}

\section{Distribuição Gama tipo III Weibull Generalizada}

A distribuição Weibull é uma distribuição de probabilidade contínua. É nomeada devido a Waloddi Weibull que, em 1951, publicou um artigo descrevendo a distribuição em detalhes e propondo diversas aplicações. O campo de aplicações da distribuição Weibull é vasto e abrange praticamente todas as áreas da ciência. Usando esta distribuição, realizou-se a modelagem bem sucedida de dados provenientes de grandes áreas das ciências física, biológica, social, saúde e ambiental. Métodos baseados nesta distribuição são ferramentas indispensáveis para profissionais da engenharia que trabalham com confiabilidade. Em geral, suas aplicações visam a determinação do tempo de vida médio e da taxa de falhas em função do tempo. É também de grande interesse para estatísticos devido às suas diversas características específicas. O sucesso da distribuição se justifica não só por sua flexibilidade, mas também pelo fato de existirem recursos gráficos que facilitam sua interpretação e por ser capaz de proporcionar previsões com acurácia razoável, mesmo quando a quantidade de dados disponível é baixa. Uma variável aleatória $X$ segue a distribuição Weibull se sua função de distribuição acumulada é dada por

$$
G_{k, v}(x)=1-e^{-(x / v)^{k}}, x>0
$$


onde $k>0$ e $v>0$ são os parâmetros de forma e de escala, respectivamente. A função densidade de probabilidade da distribuição Weibull é

$$
g_{k, v}(x)=\frac{k}{v}\left(\frac{x}{v}\right)^{k-1} e^{-\left(\frac{x}{v}\right)^{k}}, x>0 .
$$

Define-se a distribuição GIIIWG, com quatro parâmetros, tomando $G(x)$, na equação (1.1), como sendo a fda (3.1) da distribuição Weibull. Então a distribuição acumulada da GIIIWG torna-se

$$
F(x)=I_{1-e^{-(x / v)^{k}}}(a, r)=\frac{1}{\Gamma(a)} \int_{0}^{\left[e^{(x / v)^{k}}-1\right]^{r}} t^{a-1} e^{-t} d t, \quad x>0,
$$

onde $a>0, r>0, k>0$ e $v>0$. A função densidade e a função de taxa de falha correspondentes à equação (3.3) são

$$
f(x)=\frac{r}{\Gamma(a)} \frac{k}{v}\left(\frac{x}{v}\right)^{k-1}\left[1-e^{-(x / v)^{k}}\right]^{r a-1} \exp \left\{r a(x / v)^{k}-\left[e^{(x / v)^{k}}-1\right]^{r}\right\}, x>0,
$$

$\mathrm{e}$

$$
h(x)=\frac{r}{\Gamma(a)} \frac{k}{v}\left(\frac{x}{v}\right)^{k-1} \frac{\left(1-e^{-(x / v)^{k}}\right)^{r a-1}}{\left[1-I_{1-e^{-(x / v)^{k}}}(a, r)\right]} \exp \left\{r a(x / v)^{k}-\left[e^{(x / v)^{k}}-1\right]^{r}\right\}, x>0,
$$

respectivamente. Se $X$ é uma variável aleatória com densidade, então escrevemos $X \sim \operatorname{GIIIWG}(a, r, k, v)$. A função densidade de GIIIWG (3.4) inclui três submodelos importantes: a distribuição Gama tipo III Weibull (GIIIW) surge tomando $r=1$, a distribuição Gama tipo III Exponencial Generalizada (GIIIEG) surge quando $k=1$ e a distribuição Gama tipo III Exponencial (GIIIE) que surge quando $r=k=1$.

Gráficos da densidade (3.4) e da função de taxa de falha (3.5) para valores selecionados de $a, r, k, v$ são dados nas Figuras 3.1, 3.2 e nas Figuras 3.3, 3.4; respectivamente. O parâmetro r de forma extra da GIIIWG fornece maior flexibilidade na forma da distribuição gerada, por conseguinte, é mais útil para a modelagem de dados positivos. Equações laboriosas são necessárias para alcançar formatos de densidade como apresentados nas Figuras 3.1(e), 3.1(f) e 3.2(b), em especial, o formato do gráfico da Figura 3.2(b) com $a=3,5$. Pode-se notar nas Figuras 3.3 e 3.4 que as taxas de falha do novo modelo assumem as formas de banheira, monotonicamente decrescente ou crescente, dependendo dos valores dos parâmetros. Os formatos de banheira da taxa 
de falha tais como são apresentados nas Figuras 3.3(a), 3.3(c), 3.3(d), 3.3(e) e 3.4(a) são custosos de ser obtidos, implicando um grande potencial para a nova distribuição GIIIWG.

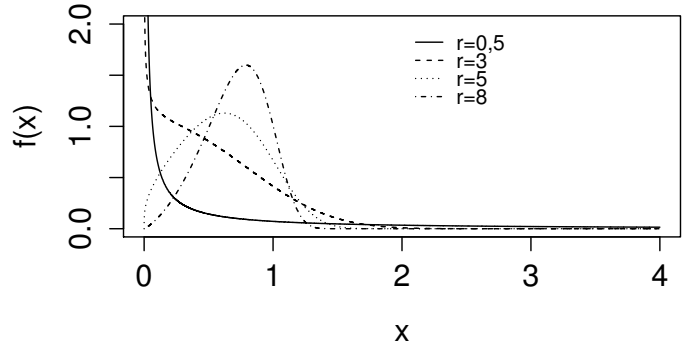

(a) $\operatorname{GIIIWG}(a=0,5, r, k=0,5, v=2)$

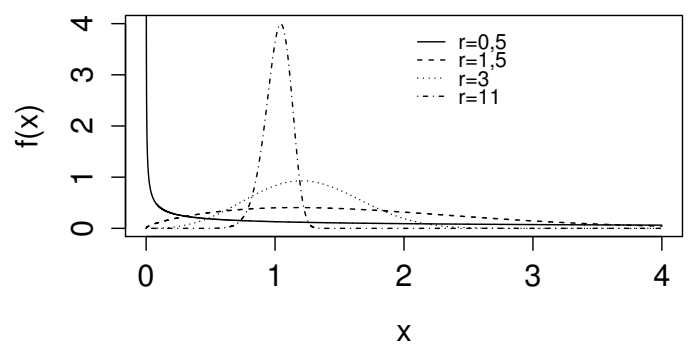

(c) $\operatorname{GIIIWG}(a=2, r,=0,5, v=2)$

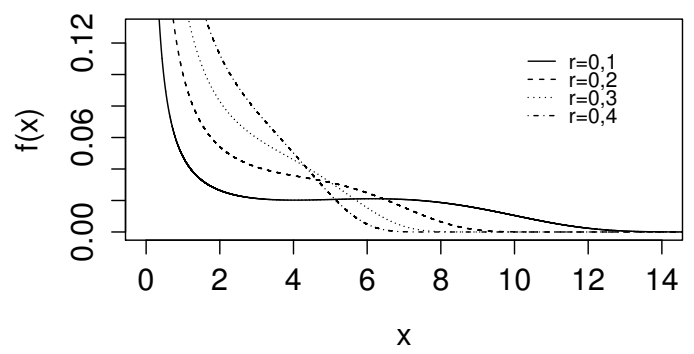

(e) $\operatorname{GIIIWG}(a=0,5, r, k=2, v=3)$

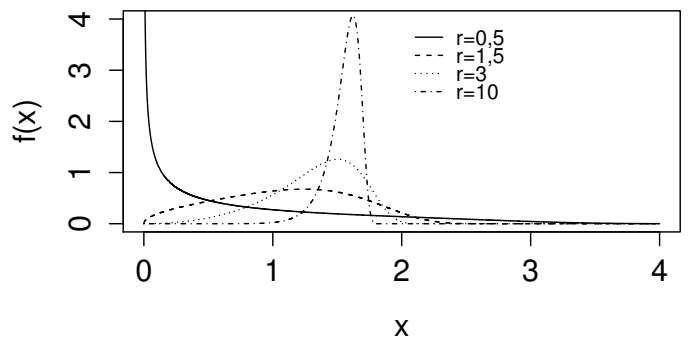

(b) GIIIWG $(a=0,5, r, k=2, v=2)$

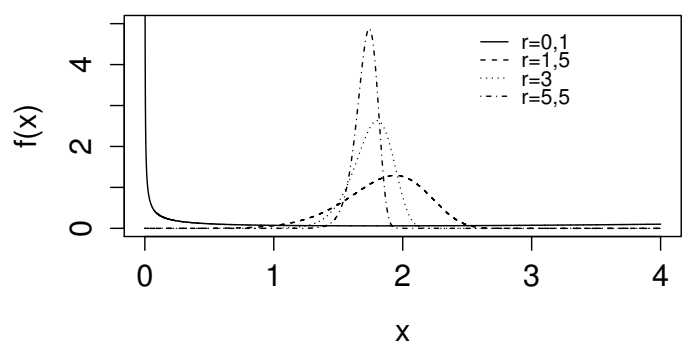

(d) $\operatorname{GIIIWG}(a=2, r, k=2, v=2)$

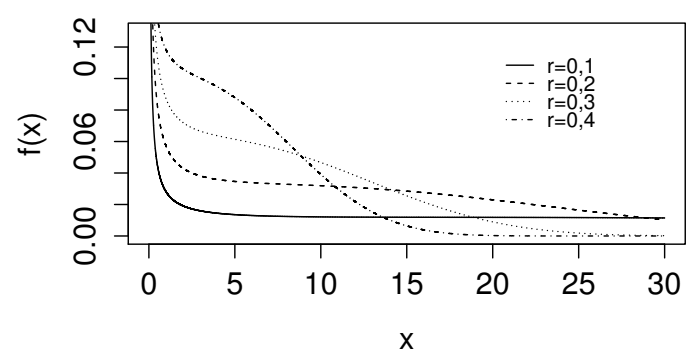

(f) $\operatorname{GIIIWG}(a=2,5, r, k=0,7, v=1,5)$

Figura 3.1: Gráficos da função densidade GIIIWG para alguns valores do parâmetro de forma $r$ em seis cenários diferentes. 


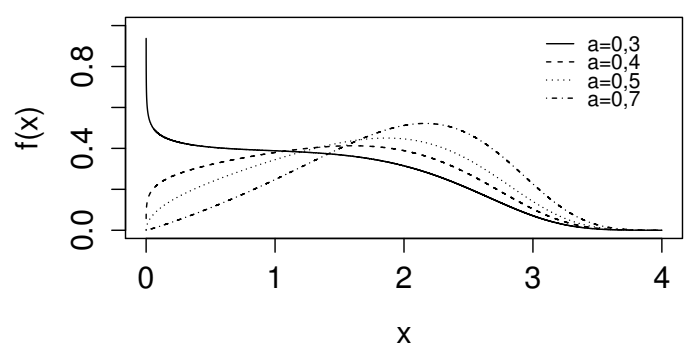

(a) $\operatorname{GIIIWG}(a, r=1,5, k=2, v=3)$

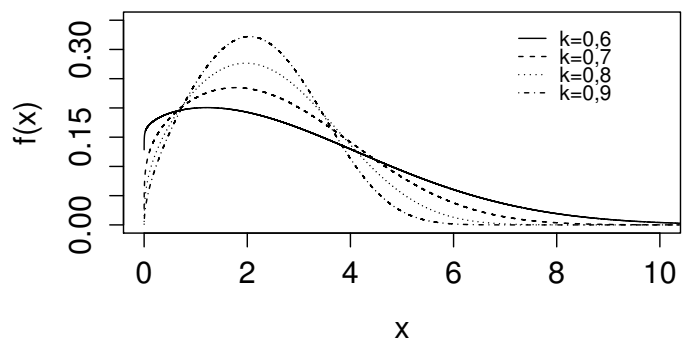

(c) $\operatorname{GIIIWG}(a=2,5, r=0,7, k, v=1,5)$

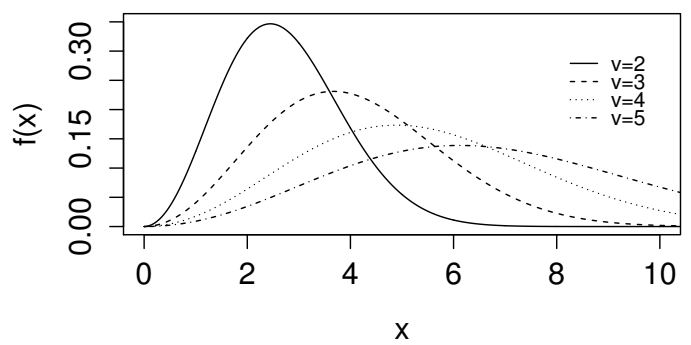

(e) GIIIWG $(a=3,5, r=1,5, k=0,5, v)$

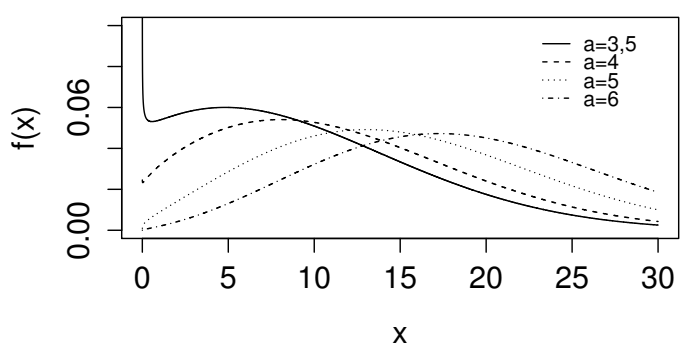

(b) $\operatorname{GIIIWG}(a, r=0,5, k=0,5, v=1,5)$

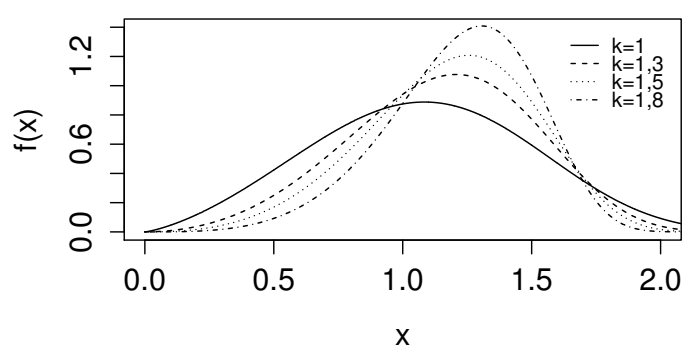

(d) $\operatorname{GIIIWG}(a=1,4, r=1,6, k, v=1,5)$

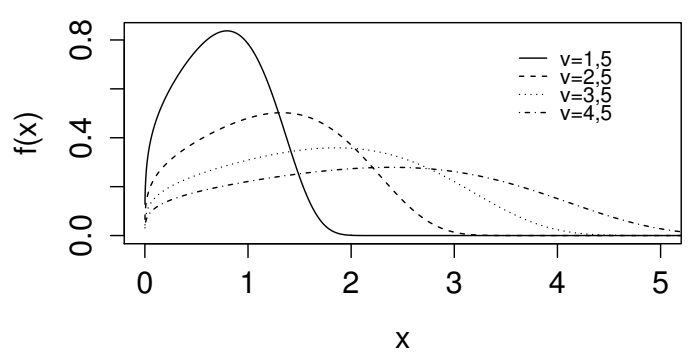

(f) $\operatorname{GIIIWG}(a=0,5, r=1,5, k=1,7, v)$

Figura 3.2: Gráficos da função densidade GIIIWG para alguns valores dos parâmetros de escala $v$ e de forma $a, k$, cada um em dois cenários diferentes. 


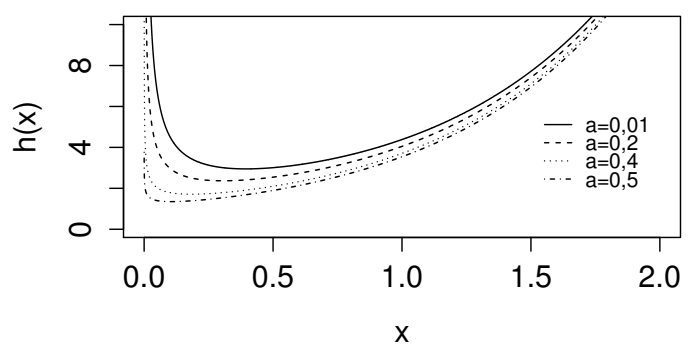

(a) $\operatorname{GIIIWG}(a, r=1,5, k=1,1, v=1,3)$

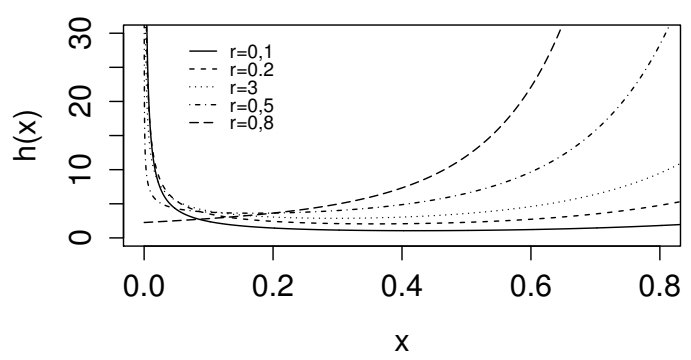

(c) $\operatorname{GIIIWG}(a=0,5, r, k=2,5, v=0,5)$

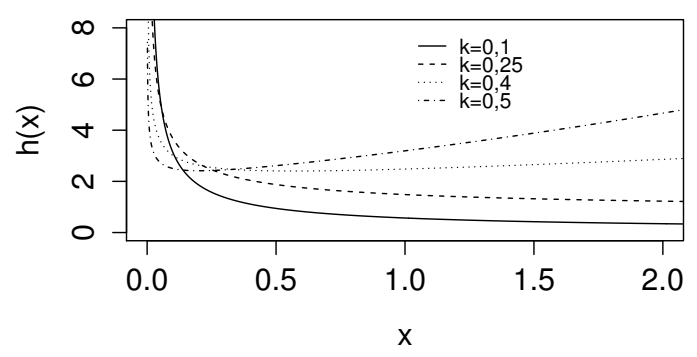

(e) $\operatorname{GIIIWG}(a=0,9, r=1,5, k, v=0,9)$

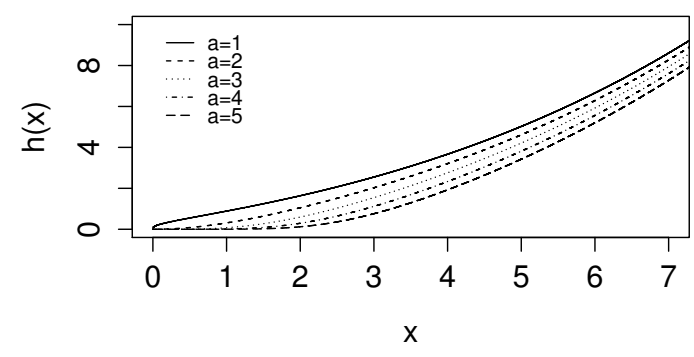

(b) $\operatorname{GIIIWG}(a, r=2,5, k=0,5, v=3)$

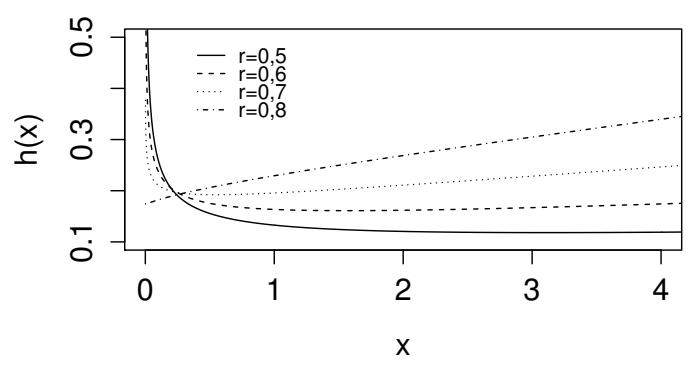

(d) $\operatorname{GIIIWG}(a=2,5, r, k=0,5, v=1,7)$

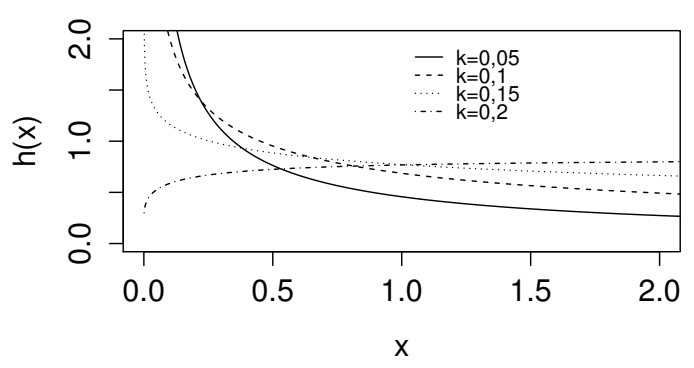

(f) $\operatorname{GIIIWG}(a=2, r=2,5, k, v=3)$

Figura 3.3: Gráficos da função taxa de falha de GIIIWG para alguns valores dos parâmetros de forma $a, r, k$, cada um em dois cenários diferentes. 


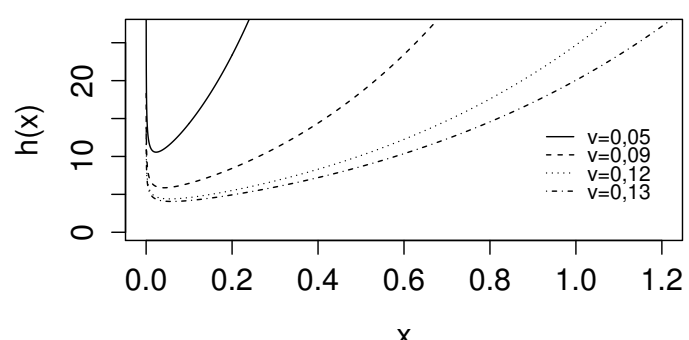

(a) $\operatorname{GIIIWG}(a=1,9, r=0,6, k=0,7, v)$

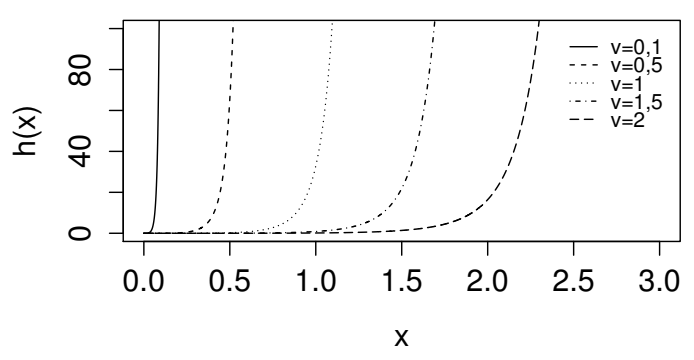

(b) $\operatorname{GIIIWG}(a=0,8, r=1,9, k=3,7, v)$

Figura 3.4: Gráficos da função taxa de falha de GIIIWG para alguns valores do parâmetro de escala $v$ em dois cenários diferentes.

\subsection{Expansão da função densidade e momentos}

Se G é a fda da variável aleatoria Weibull (3.1) com parâmetros $k$ e $v$, então

$$
G_{k, v}(x)^{r(a+j)+i}=\left[1-e^{-(x / v)^{k}}\right]^{r(a+j)+i} .
$$

Ou seja, $G_{k, v}(x)^{r(a+j)+i}$ é a fda da distribuição Weibull exponenciada.

Considerando a expansão

$$
(1-x)^{b}=\sum_{h=0}^{\infty}(-1)^{h} \frac{\Gamma(b+1)}{\Gamma(b+1-h) h !} x^{h}, \text { para } b>0,0<x<1,
$$

e que $0<e^{-(x / v)^{k}}<1$, pois $x, k, v>0$, tem-se que pela equação (1.6)

$$
\begin{aligned}
F(x) & =\sum_{j=0}^{\infty} \sum_{i=0}^{\infty} \omega_{j, i} G(x)^{r(a+j)+i} \\
& =\sum_{j=0}^{\infty} \sum_{i=0}^{\infty} \omega_{j, i}\left[1-e^{-(x / v)^{k}}\right]^{r(a+j)+i} \\
& \stackrel{(3.6)}{=} \sum_{j=0}^{\infty} \sum_{i=0}^{\infty} \omega_{j, i} \sum_{h=0}^{\infty}(-1)^{h} \frac{\Gamma(r(a+j)+i+1)}{\Gamma(r(a+j)+i+1-h) h !}\left[e^{-(x / v)^{k}}\right]^{h} \\
& =\sum_{j=0}^{\infty} \sum_{i=0}^{\infty} \omega_{j, i}\left\{1+\sum_{h=1}^{\infty}(-1)^{h} \frac{\Gamma(r(a+j)+i+1)}{\Gamma(r(a+j)+i+1-h) h !} e^{-\left(h^{1 / k} x / v\right)^{k}}\right\} \\
& =\sum_{j=0}^{\infty} \sum_{i=0}^{\infty} \omega_{j, i}\left\{1+\sum_{h=1}^{\infty}(-1)^{h} \frac{\Gamma(r(a+j)+i+1)}{\Gamma(r(a+j)+i+1-h) h !}\left[1-G_{k, \frac{v}{h^{1 / k}}}(x)\right]\right\} .
\end{aligned}
$$


Logo,

$$
\begin{aligned}
f(x) & =\sum_{j=0}^{\infty} \sum_{i=0}^{\infty} \sum_{h=1}^{\infty} \omega_{j, i}(-1)^{h+1} \frac{\Gamma(r(a+j)+i+1)}{\Gamma(r(a+j)+i+1-h) h !} g_{k, \frac{v}{h^{1 / k}}}(x) \\
& =\sum_{j=0}^{\infty} \sum_{i=0}^{\infty} \sum_{h=1}^{\infty} \omega_{j, i, h} g_{k, \frac{v}{h^{1 / k}}}(x),
\end{aligned}
$$

onde

$$
\omega_{j, i, h}=\omega_{j, i}(-1)^{h+1} \frac{\Gamma(r(a+j)+i+1)}{\Gamma(r(a+j)+i+1-h) h !}
$$

e $g_{k, \frac{v}{h^{1 / k}}}(x)$ é a fdp da Weibull com parâmetros $k, \frac{v}{h^{1 / k}}$. Como o r-ésimo momento da distribuição Weibull com parâmetros $k$ e $v$ é $\mu_{r}^{\prime}=\Gamma(r / k+1) v^{r}$,

$$
E\left(X^{r}\right)=\sum_{j=0}^{\infty} \sum_{i=0}^{\infty} \sum_{h=1}^{\infty} \omega_{j, i, h} \Gamma(r / k+1)\left(\frac{v}{h^{1 / k}}\right)^{r} .
$$

Gráficos de assimetria e curtose para valores selecionados de $a$ como função de $r$, para algumas escolhas de $r$ como função de $a$, para algumas escolhas de $k$ como função de $r$, e para algumas escolhas de $r$ como função de $k$, são mostradas nas Figuras 3.5 e 3.6.

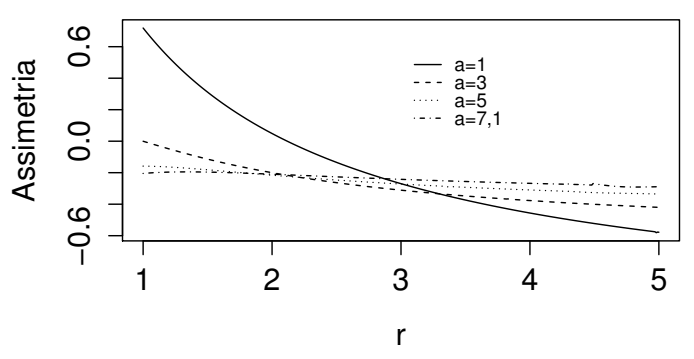

(a) $\operatorname{GIIIWG}(a, r, k=1, v=1)$

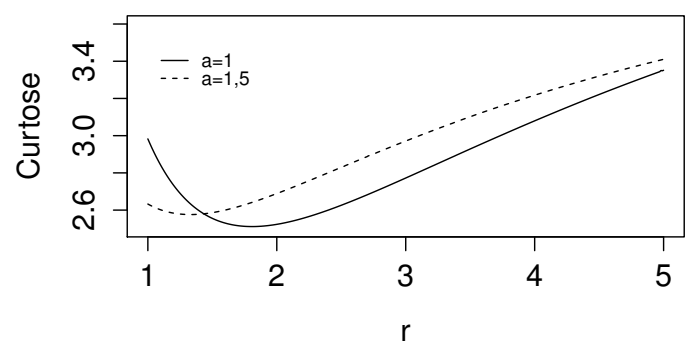

(b) GIIIWG $(a, r, k=1, v=1)$

Figura 3.5: Assimetria e curtose da distribuição GIIIWG como função de $a$ e $r$. 


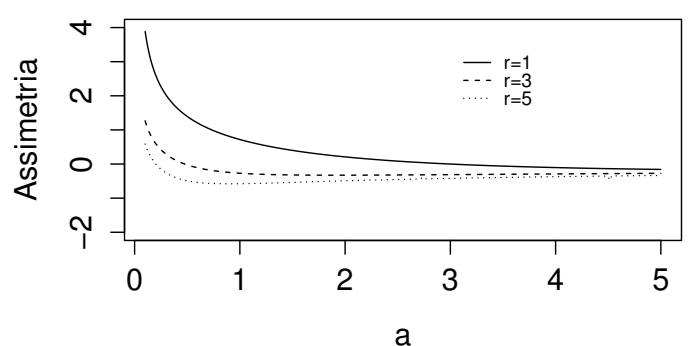

(a) $\operatorname{GIIIWG}(a, r, k=1, v=1)$

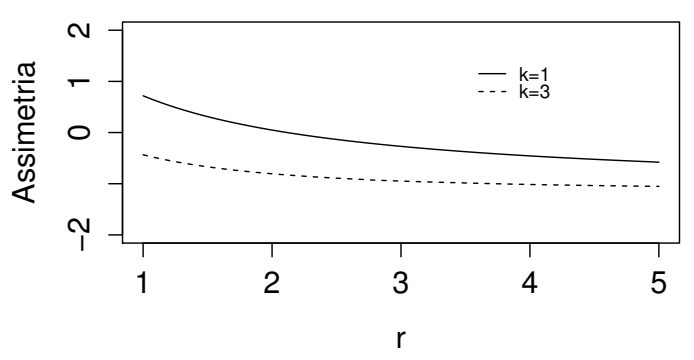

(c) $\operatorname{GIIIWG}(a=1, r, k, v=1)$

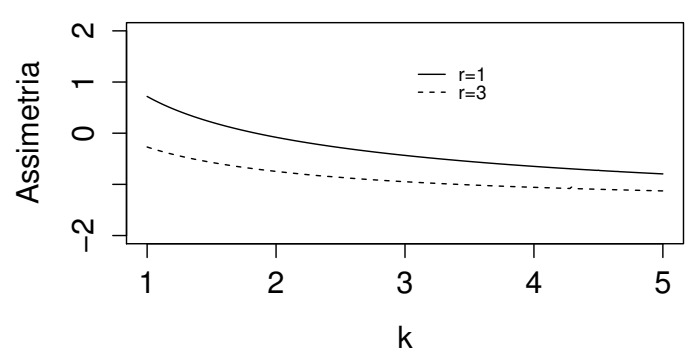

(e) $\operatorname{GIIIWG}(a=1, r, k, v=1)$

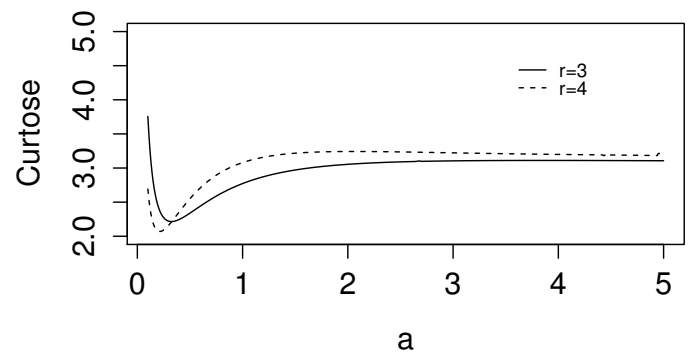

(b) $\operatorname{GIIIWG}(a, r, k=1, v=1)$

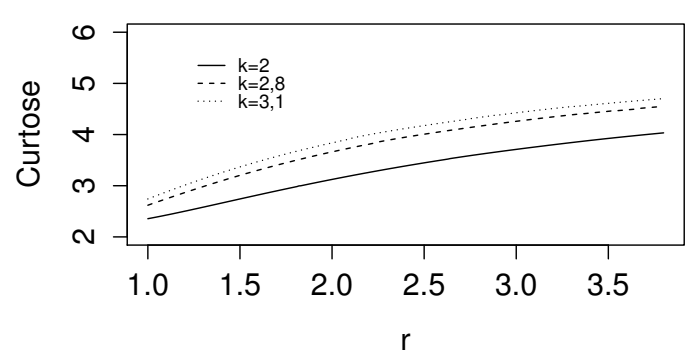

(d) $\operatorname{GIIIWG}(a=1, r, k, v=1)$

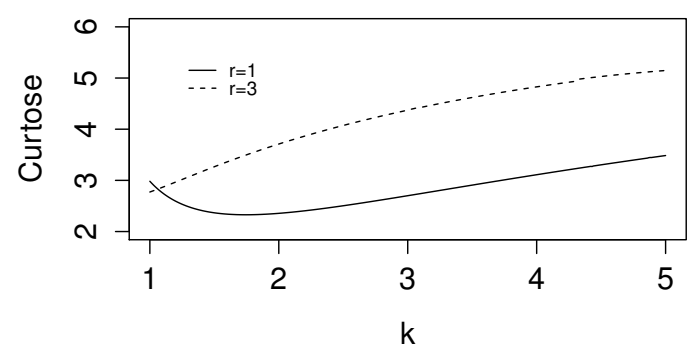

(f) $\operatorname{GIIIWG}(a=1, r, k, v=1)$

Figura 3.6: Assimetria e curtose da distribuição GIIIWG como função de $a$ e $r, k$ e $r$. 


\subsection{Função quantílica e simulação}

Seja G a fda da distribuição Weibull (3.1), sua inversa é dada por

$$
G_{k, v}^{-1}(y)=v[-\log (1-y)]^{1 / k}
$$

Com isso, usando a Equação (1.7), tem-se que

$$
x=v\left[-\log \left(1-\frac{\left[Q_{a, 1}(u)\right]^{1 / r}}{1+\left[Q_{a, 1}(u)\right]^{1 / r}}\right)\right]^{-1 / k} .
$$

Consequentemente, pode-se gerar a variável aleatória GIIIWG pela Equação (3.7), onde $u$ é gerada da distribuição Uniforme $(0,1)$ e $Q_{a, 1}(u)$ está disponível na maioria dos pacotes dos softwares estatísticos.

\subsection{Aplicação}

Com um conjunto de dados reais, nesta seção, fornecemos uma aplicação que prova a flexibilidade do modelo GIIIWG.

Para o modelo GIIIWG dado pela equação (3.4) trabalhamos com os dados que representam o tempo até a falha de 50 dispositivos colocados no teste de vida no tempo 0, observado e reportado por Aarset [26]. Os dados são como seguem:

$0,10,2111112367111218181818182132364045464750556063$ 636767676772757982828384848485858585858686 .

Ajustamos as distribuições GIIIWG, GIIIW, GIIIEG e GIIIE aos dados pelo método de máxima verossimilhança. Os EMV's dos parâmetros, com erros padrões (EP), medidos para os modelos são mostrados na Tabela 3.1. O teste de hipótese é dado na Tabela 3.2 .

As estatísticas RV para testar as hipóteses $H_{0}$ : GIIIW $\times H_{A}$ : GIIIWG, $H_{0}$ : GIIIEG $\times H_{A}$ : GIIIWG e $H_{0}:$ GIIIE $\times H_{A}$ : GIIIWG são 82, 991 (p-valor=0), 19, 391 $\left(\mathrm{p}\right.$-valor $\left.=1 \times 10^{-5}\right)$ e $33,247\left(\mathrm{p}\right.$-valor $\left.=6 \times 10^{-8}\right)$, respectivamente. Com isso, rejeitamos a hipótese nula em todos os casos à favor da distribuição GIIIWG. Gráficos das densidades ajustadas das distribuições GIIIWG, GIIIW, GIIIEG e GIIIE são dados na Figura 3.7. O gráfico indica que a distribuição GIIIWG fornece um melhor ajuste para estes dados do que seus submodelos. É importante notar que, na Figura 
3.7, o parâmetro $r$ quebra a convexidade estrita das distribuições GIIIW e GIIIE, tornando-as parcialmente unimodais como as distribuições GIIIWG e GIIIEG. Fatigantes equações são necessárias para se obter o formato de densidade ajustado pela GIIIWG e GIIIEG como neste caso, demonstrando, mais uma vez, um grande potencial da nova distribuição. As implementações, usando o programa R, das estimativas estão no Apêndice.

\begin{tabular}{ccccc}
\hline Modelo & $\mathrm{a}$ & $\mathrm{r}$ & $k$ & $v$ \\
\hline GIIIWG & 1,519 & 0,118 & 3,299 & 42,453 \\
& $(0,755)$ & $(0,061)$ & $(1,334)$ & $(17,846)$ \\
GIIIW & 2,687 & 1 & 0,133 & 1,767 \\
& $(0,015)$ & $(-)$ & $(0,192)$ & $(0,148)$ \\
GIIIEG & 2,442 & 0,198 & 1 & 11,515 \\
& $(0,372)$ & $(0,057)$ & $(-)$ & $(3,689)$ \\
GIIIE & 0,743 & 1 & 1 & 86,784 \\
& $(0,125)$ & $(-)$ & $(-)$ & $(7,097)$ \\
\hline
\end{tabular}

Tabela 3.1: Estimativas de máxima verossimilhança dos parâmetros dos modelos GIIIWG, GIIIW, GIIIEG e GIIIE.

\begin{tabular}{cccc}
\hline Modelo & Hipótese & Estatística $\mathrm{w}$ & $\mathrm{p}$-valor \\
\hline GIIIWG x GIIIW & $H_{0}: r=1$ & 82,991 & 0 \\
GIIIWG x GIIIEG & $H_{0}: k=1$ & 19,391 & $1 \times 10^{-5}$ \\
GIIIWG x GIIIE & $H_{0}: r=k=1$ & 33,247 & $6 \times 10^{-8}$ \\
\hline
\end{tabular}

Tabela 3.2: Testes de Hipóteses dos modelos. 


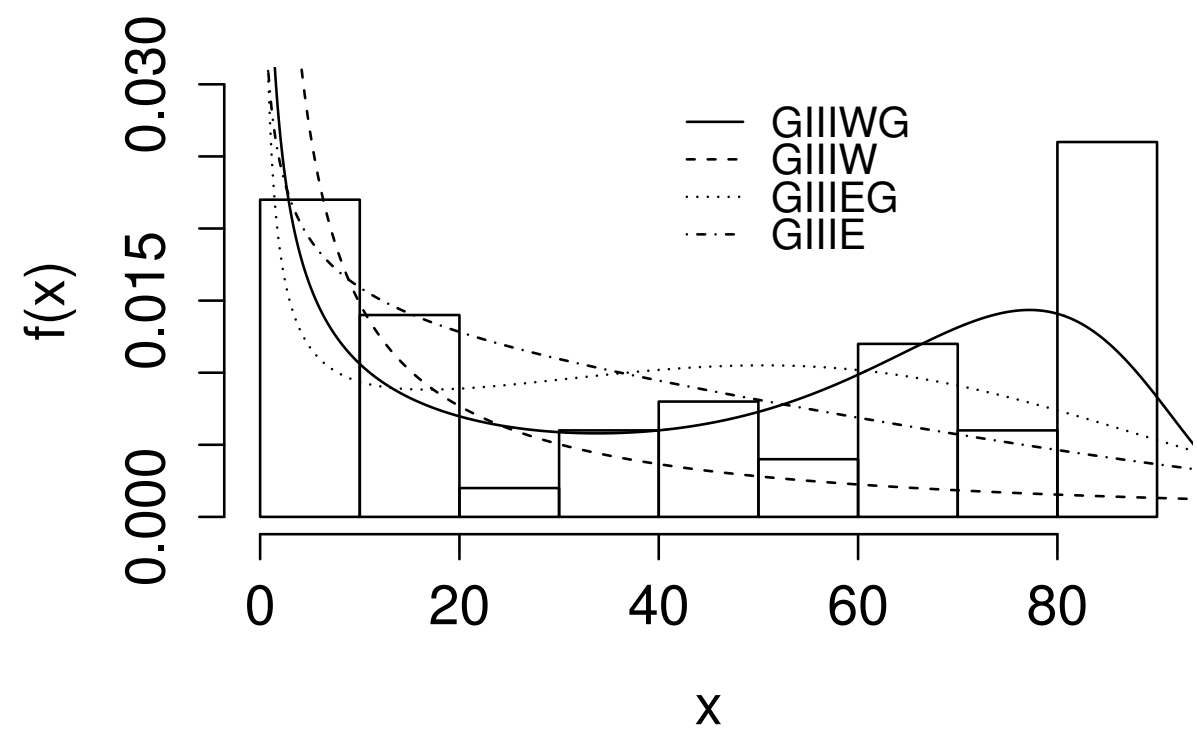

Figura 3.7: Gráfico dos modelos GIIIWG, GIIIW, GIIIEG e GIIIE ajustados a um conjunto de dados reais com seu histograma. 


\section{Capítulo 4}

\section{Conclusões e Trabalhos Futuros}

\subsection{Conclusões}

Neste trabalho, propomos e estudamos as propriedades de uma nova família de distribuições, a família Gama tipo III Generalizada. Esta família é uma generalização da família Gama tipo III proposta por Torabi e Montazeri [6]. Estudamos expansões da função densidade de probabilidade e da função de distribuição acumulada, os momentos, a função quantílica e a entropia, além do ajuste do modelo a dados reais. Com o intuito de avaliar a flexibilidade desta família, estudamos dois de seus modelos: a Gama tipo III Burr III Generalizada (GIIIBIIIG) e a Gama tipo III Weibull Generalizada (GIIIWG). Os gráficos da função densidade de probabilidade e da função taxa de falha evidenciam a grande flexibilidade destes modelos para a descrição de dados. Estes modelos foram ajustados a dois conjuntos de dados reais e se mostraram superiores a seus submodelos na descrição do comportamento dos dados. 


\subsection{Trabalhos Futuros}

Este trabalho evidenciou o grande potencial da família de distribuições Gama tipo III Generalizada. Pretendemos estudar as propriedades de outros modelos desta família não abordados aqui. Pretendemos, também, estudar a utilização de modelos desta família em modelos de regressão. 


\section{Referências Bibliográficas}

[1] Mudholkar GS, Srivastava, DK. Exponentiated Weibull Family for Analyzing Bathtub Failure-Rate Data. IEEE Transations on Reliability. 1993;42:299-302.

[2] Gupta RD, Kundu D. Generalized Exponential Distributions. Australian and New Zealand Journal of Statistis. 1999;41:173-188.

[3] Eugene N, Lee C, Famoye F. Beta-normal distribution and its applications. Communication in Statistics-Theory and Methods. 2002;31:497-512.

[4] Stacy EW. A generalization of the gamma distribution. Annals of Mathematical Statistics. 1962;33:1187-1192.

[5] Cordeiro GM, Castro M. A new family or generalized distributions. Journal of Statistical Computation and Simulation. 2011;81:827-842.

[6] Torabi H, Montazeri NH. The gamma-uniform distribution and its applications. Kybernetika. 2012;48:16-30.

[7] Tahir MH, Cordeiro GM, Mansoor M, Zubair M, Alizadeh M. The WeibullDagum Distribution: Properties and Applications. Communications in StatisticsTheory and Methods; 2016. doi:10.1080/03610926.2014.983610.

[8] Burr IW. Cumulative frequency distributions. Annals of Mathematical Statistics. $1942 ; 13: 215-232$.

[9] Dagum, C. A new model of personal income distribution: specification and estimation. Economie Apliqueé. 1977;30:413-437.

[10] Dagum C. The generation and distribution of income, the Lorenz curve and the Gini ration. Economie Apliqueé. 1980;33:327-367. 
[11] Kleiber C, Kotz S. Statistical size distribuition in economics and actuarial sciences. New York: John Wiley \& Sons, Inc.; 2003.

[12] Kleiber C. A guide to the Dagum distribution. In: Duangkamon C, editor. Modeling income distributions and Lorenz curves series: economic studies in inequality, social exclusion and well-being, vol. 5. New York: Springer; 2008.

[13] Klugman SA, Panjer HH, Willmot GE. Loss models. New York: John Wiley; 1998.

[14] Mielke PW. Another family of distributions for describing and analyzing precipitation data. J Appl Meterol. 1973;12:275-280.

[15] Mielke PW, Johnson ES. Three-parameter kappa distribution maximum likelihood estimates and likelihood ratio test. Mon Weather Rev. 1973;101:701-707.

[16] Sherrick BJ, Garcia P, Tirupattur V. Recovering probabilistic information from option markets: test of distributional assumptions. J Fut Mark. 1996;16:545-560.

[17] Lindsay SR, Wood GR, Woollons RC. Modelling the diameter distribution of forest stands using the Burr distribution. J Appl Stat. 1996;23:609-619.

[18] Al-Dayian GR. Burr type III distribution: properties and estimation. Egypt Stat J. 1999;43:102-116.

[19] Shao Q. Estimation for hazardous concentrations based on NOEC toxicity data: an alternative approach. Environmetrics. 2000;11:583-595.

[20] Hose GC. Assessing the need for groundwater quality guidelines for pesticides using the species sensitivity distribution approach. Hum Ecol Risk Assess. 2005;11:951-966.

[21] Mokhlis NA. Reliability of a stress-strength model with Burr type III distributions. Commun Stat Theory Methods. 2005;34:1643-1657.

[22] Gove JH, Ducey MJ, Leak WB, Zhang L. Rotated sigmoid structures in managed uneven-aged northern hardwood stands: a look at the Burr type III distribution. Forestry; 2008. doi:10.1093/forestry/cpm025. 
[23] Shao Q, Chen YD, Zhang L. An extension of three-parameter Burr III distribution for low-flow frequency analysis. Comput Stat Data Anal. 2008;52:1304-1314.

[24] Rodriguez RN. Multivariate Burr III distributions, Part I. Theoretical properties. Warren, MI: General Motors Research Laboratories; 1980 [Research Publication GMR-3232].

[25] Bjerkedal T. Acquisition of Resistance in Guinea Pies infected with Different Doses of Virulent Tubercle Bacilli. American Journal of Hygiene. 1960;72(1):130148.

[26] Aarset MV. How to Identify a Bathtub Hazard Rate, IEEE Transactions on Reliability. 1987;36(1):106-108. 


\section{Apêndice 1}

\#\#\# Programação da GIIIBIIIG\#\#

\# fdp e fda da BIII:

pburr<-function $(\mathrm{x})\left\{\left(\left((\mathrm{x} / \mathrm{s})^{\wedge}\right.\right.\right.$ alpha $) /(1+$

$(\mathrm{x} / \mathrm{s})^{\wedge} \mathrm{al}$ pha $\left.)\right)^{\wedge}$ beta\}

dburr $<-$ function $(x)\{$ alpha*beta* $((((x / s) \wedge a l p h a) /(1+$

$\left.\left.\left.\left.(\mathrm{x} / \mathrm{s})^{\wedge} \mathrm{alpha}\right)\right)^{\wedge}(\operatorname{beta}+1)\right) /\left(\mathrm{s} *(\mathrm{x} / \mathrm{s})^{\wedge}(\mathrm{alpha}+1)\right)\right\}$

\# fdp da GIIIBIIIG:

$\mathrm{fdp}<-$ function $(\mathrm{x})\left\{(\mathrm{r} * \operatorname{dburr}(\mathrm{x}) / \operatorname{gamma}(\mathrm{a})) *\left(\operatorname{pburr}(\mathrm{x})^{\wedge}(\mathrm{r} * \mathrm{a}-1)\right) /\right.$ $\left((1-\operatorname{pburr}(\mathrm{x}))^{\wedge}(\mathrm{r} * \mathrm{a}+1)\right) *$

$\left.\exp \left(-(\operatorname{pburr}(\mathrm{x}) /(1-\operatorname{pburr}(\mathrm{x})))^{\wedge} \mathrm{r}\right)\right\}$

\# Gráficos da fdp da GIIIBIIIG:

\# a1

$r=1$

alpha $=0.9$

beta $=0.9$

$\mathrm{s}=0.5$

\#abcissa

$\mathrm{x}<-\operatorname{seq}(0.01,4,0.01)$

\#linha 1

$\mathrm{a}=1.15$

$\mathrm{y}<-\mathrm{fdp}(\mathrm{x})$

plot $(x, y$, type $=" 1 ", y l a b=c(" f(x) "))$ 
\#linha 2

$a=1.5$

$\mathrm{y}<-\mathrm{fdp}(\mathrm{x})$

lines $(x, y, l$ ty $=2)$

\#linha 3

$\mathrm{a}=2$

$y<-f d p(x)$

lines $(x, y, l$ ty $=3$ )

\#linha 4

$a=3$

$\mathrm{y}<-\mathrm{fdp}(\mathrm{x})$

lines $(x, y, l$ ty $=4)$

\#linha 5

$\mathrm{a}=5$

$y<-f d p(x)$

$\operatorname{lines}(\mathrm{x}, \mathrm{y}, 1 \mathrm{ty}=5)$

legend (2.8, 2.3, c("a=1,15", "a=1, 5" , "a=2" , "a=3", "a=5"), 1ty=c $(1,2,3,4,5)$ , bty $=" n ", c e x=.65$ )

\# a2

$r=2$

alpha $=1.5$

beta $=1.5$

$s=0.5$

\#abcissa

$x<-\operatorname{seq}(0.01,1.7,0.01)$

\#linha 5

$\mathrm{a}=3$

$\mathrm{y}<-\mathrm{fdp}(\mathrm{x})$

plot $(x, y$, type $=" 1 ", y l a b=c(" f(x) ")$, tty $=5)$

\#linha 1

$a=1.15$ 


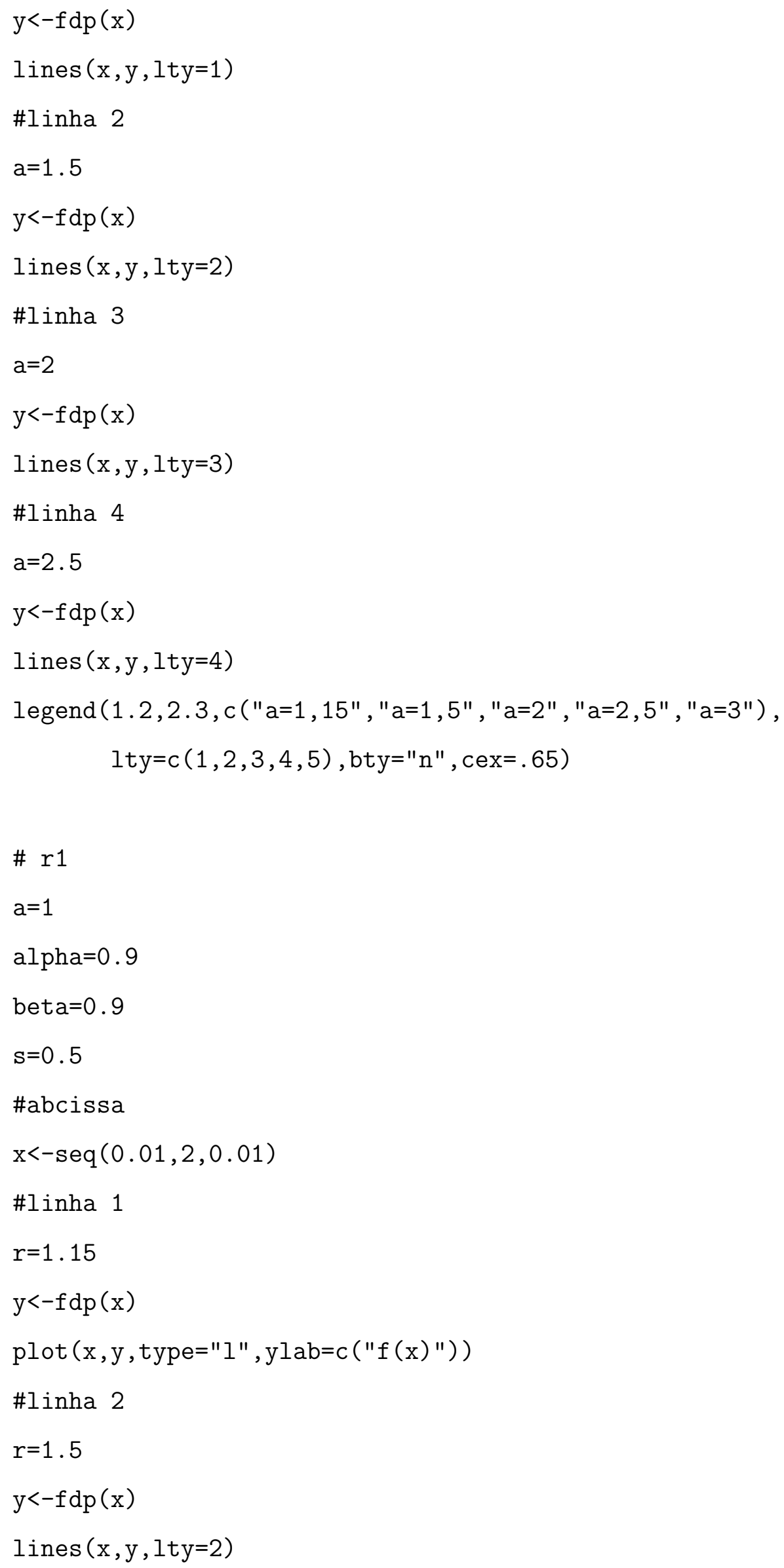


\#linha 3

$r=2$

$\mathrm{y}<-\mathrm{fdp}(\mathrm{x})$

$\operatorname{lines}(\mathrm{x}, \mathrm{y}, 1 \mathrm{ty}=3)$

\#linha 4

$r=3$

$\mathrm{y}<-\mathrm{fdp}(\mathrm{x})$

$\operatorname{lines}(\mathrm{x}, \mathrm{y}, \mathrm{lty}=4)$

legend (1.3, 2.5, c ("r=1,15", "r=1 , 5" , "r=2" , "r=3"), lty $=c(1,2,3,4)$, bty $=" n ", c e x=.65)$

\# Gráf da txf da GIIIGBIII:

\# acumulada da GIIIGBIII:

$\mathrm{fda}<-$ function $(\mathrm{x})\left\{\operatorname{pgamma}\left((\operatorname{pburr}(\mathrm{x}) /(1-\operatorname{pburr}(\mathrm{x})))^{\wedge} \mathrm{r}, \mathrm{a}, 1\right)\right\}$

\# taxa de falha:

$\mathrm{h}<-$ function $(\mathrm{x})\{\mathrm{fdp}(\mathrm{x}) /(1-\mathrm{fda}(\mathrm{x}))\}$

\# txf a1

$r=0.5$

alpha $=0.9$

beta $=0.9$

$s=0.5$

\#abcissa

$x<-\operatorname{seq}(0.001,1.5,0.001)$

\#linha 1

$a=0.09$

$\mathrm{y}<-\mathrm{h}(\mathrm{x})$

plot $(x, y$, type $=" 1 ", y l a b=c(" h(x) "), y l i m=c(0,4))$

\#linha 2

$a=1$

$\mathrm{y}<-\mathrm{h}(\mathrm{x})$

lines $(x, y, 1$ ty $=2)$ 
\#linha 3

$\mathrm{a}=2$

$\mathrm{y}<-\mathrm{h}(\mathrm{x})$

$\operatorname{lines}(\mathrm{x}, \mathrm{y}, \mathrm{lty}=3)$

\#linha 4

$a=3$

$\mathrm{y}<-\mathrm{h}(\mathrm{x})$

$\operatorname{lines}(\mathrm{x}, \mathrm{y}, \mathrm{lty}=4)$

\#linha 5

$\mathrm{a}=4$

$\mathrm{y}<-\mathrm{h}(\mathrm{x})$

lines $(x, y, 1$ ty $=5)$

legend (1, 4, c ("a=0, 09", "a=1", "a=2", "a=3" , "a=4"), 1ty=c $(1,2,3,4,5)$, bty $=" n ", c e x=0.65$ )

$\#$ txf a2

$r=2.5$

alpha $=2$

bet $a=3$

$s=0.5$

\#abcissa

$\mathrm{x}<-\operatorname{seq}(0.0001,1.5,0.0001)$

\#linha 1

$a=0.5$

$\mathrm{y}<-\mathrm{h}(\mathrm{x})$

$\operatorname{plot}(\mathrm{x}, \mathrm{y}, \mathrm{type}=" \mathrm{l} ", \mathrm{ylab}=\mathrm{c}($ "h(x)"))

\#linha 2

$a=1.5$

$\mathrm{y}<-\mathrm{h}(\mathrm{x})$

lines $(x, y, 1$ ty $=2)$

\#linha 3

$a=2.5$ 
$\mathrm{y}<-\mathrm{h}(\mathrm{x})$

lines $(x, y, 1$ ty $=3)$

\#linha 4

$\mathrm{a}=3.5$

$\mathrm{y}<-\mathrm{h}(\mathrm{x})$

lines $(x, y, 1$ ty $=4)$

\#linha 5

$\mathrm{a}=4.5$

$\mathrm{y}<-\mathrm{h}(\mathrm{x})$

lines $(x, y, 1$ ty $=5)$

legend $(0.3,40, c(" a=0,5 "$, " $a=1,5$ " , "a=2, 5" , "a=3, 5" , "a=4, 5")

, lty $=c(1,2,3,4,5)$, bty $=" n ", c e x=.65)$

\# txf r1

$\mathrm{a}=1$

alpha $=0.9$

beta $=0.9$

$s=0.5$

\#abcissa

$x<-\operatorname{seq}(0.00001,0.3,0.00001)$

\#linha 1

$r=1.13$

$\mathrm{y}<-\mathrm{h}(\mathrm{x})$

plot $(x, y$, type $=" l ", y l a b=c(" h(x) "), y l i m=c(2.15,2.8))$

\#linha 2

$r=1.15$

$\mathrm{y}<-\mathrm{h}(\mathrm{x})$

$\operatorname{lines}(\mathrm{x}, \mathrm{y}, \mathrm{lty}=2)$

\#linha 3

$r=1.17$

$\mathrm{y}<-\mathrm{h}(\mathrm{x})$

lines $(x, y, l$ ty $=3)$ 


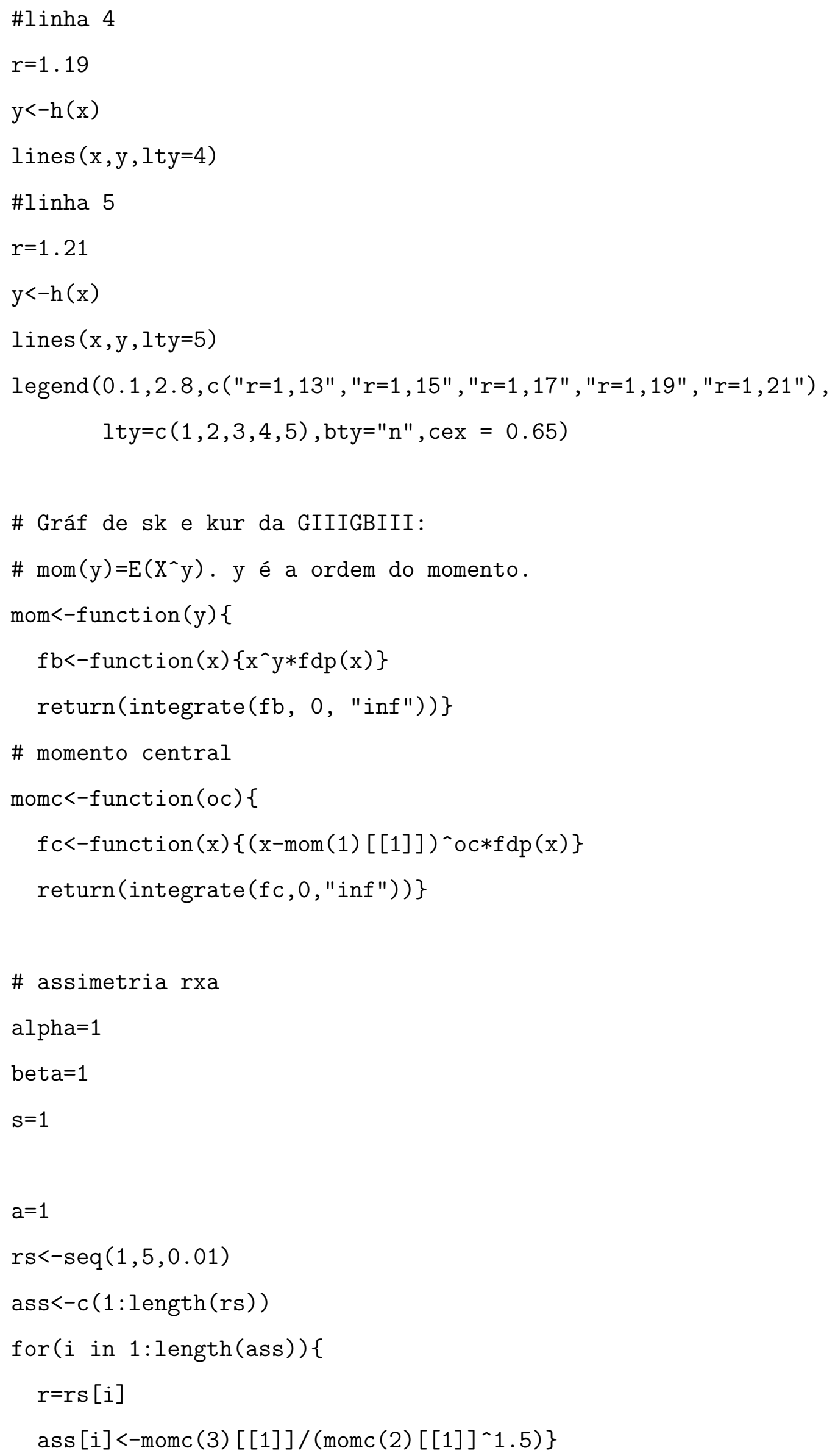




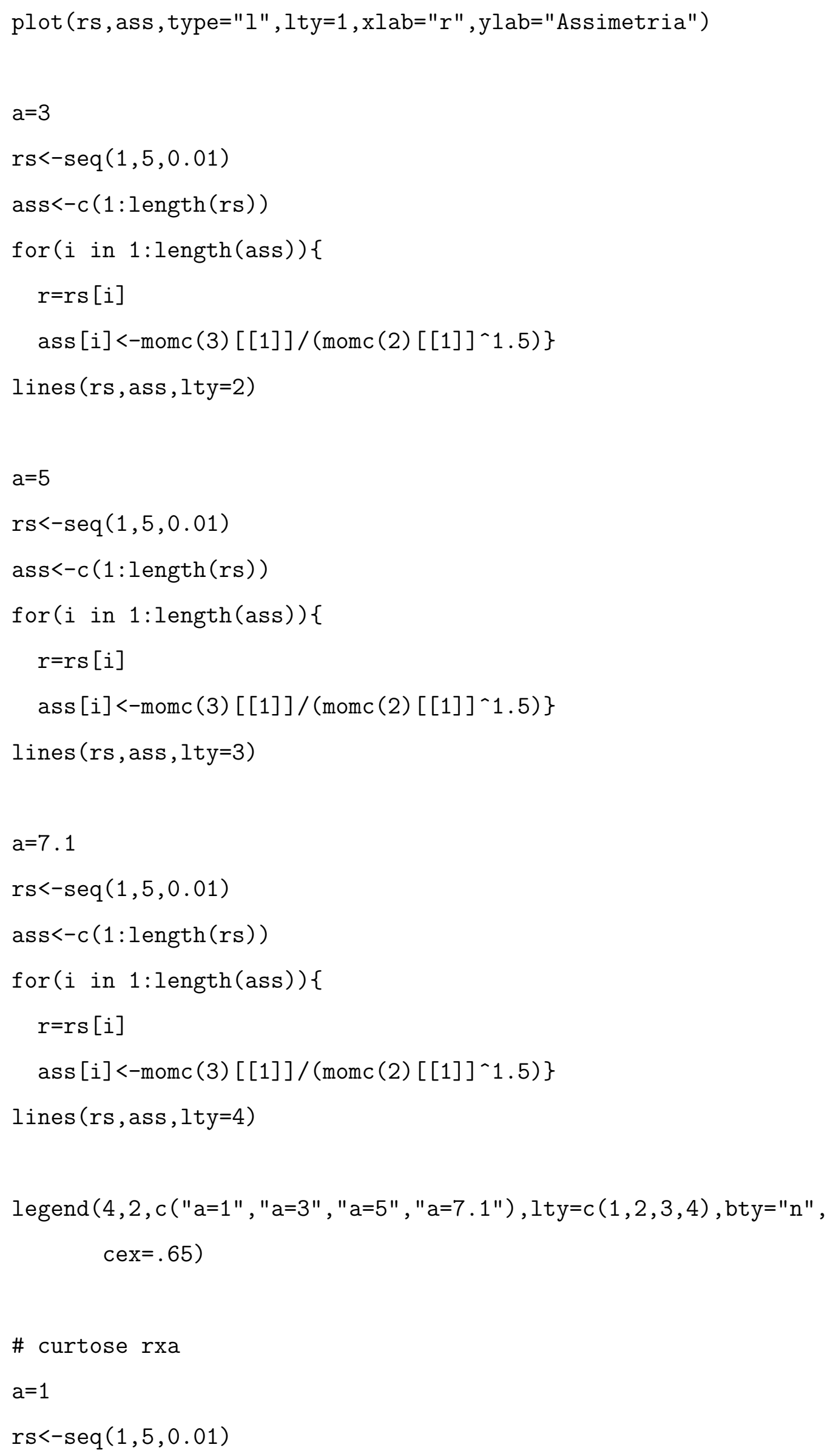




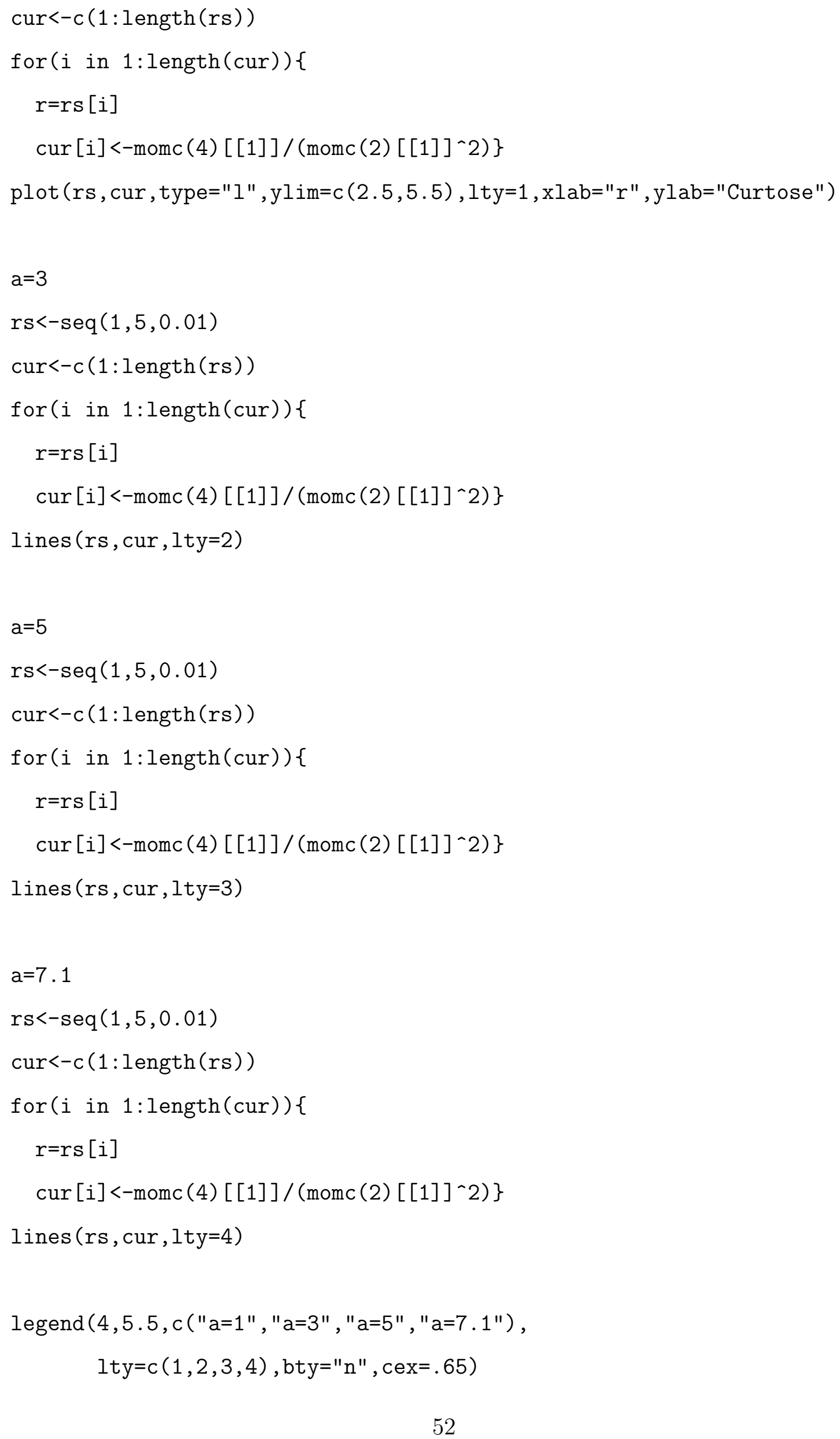


\# GIIIGBIII dados:

\# Artigo: A new generalized Lindley distribution

$\mathrm{x}<-\mathrm{c}(0.1,0.33,0.44,0.56,0.59,0.72,0.74,0.77,0.92,0.93,0.96$,

$1,1,1.02,1.05,1.07,1.07,1.08,1.08,1.08,1.09,1.12$,

$1.13,1.15,1.16,1.2,1.21,1.22,1.22,1.24,1.3,1.34,1.36$,

$1.39,1.44,1.46,1.53,1.59,1.6,1.63,1.63,1.68,1.71,1.72$,

$1.76,1.83,1.95,1.96,1.97,2.02,2.13,2.15,2.16,2.22,2.3$,

$2.31,2.4,2.45,2.51,2.53,2.54,2.54,2.78,2.93,3.27,3.42$,

$3.47,3.61,4.02,4.32,4.58,5.55)$

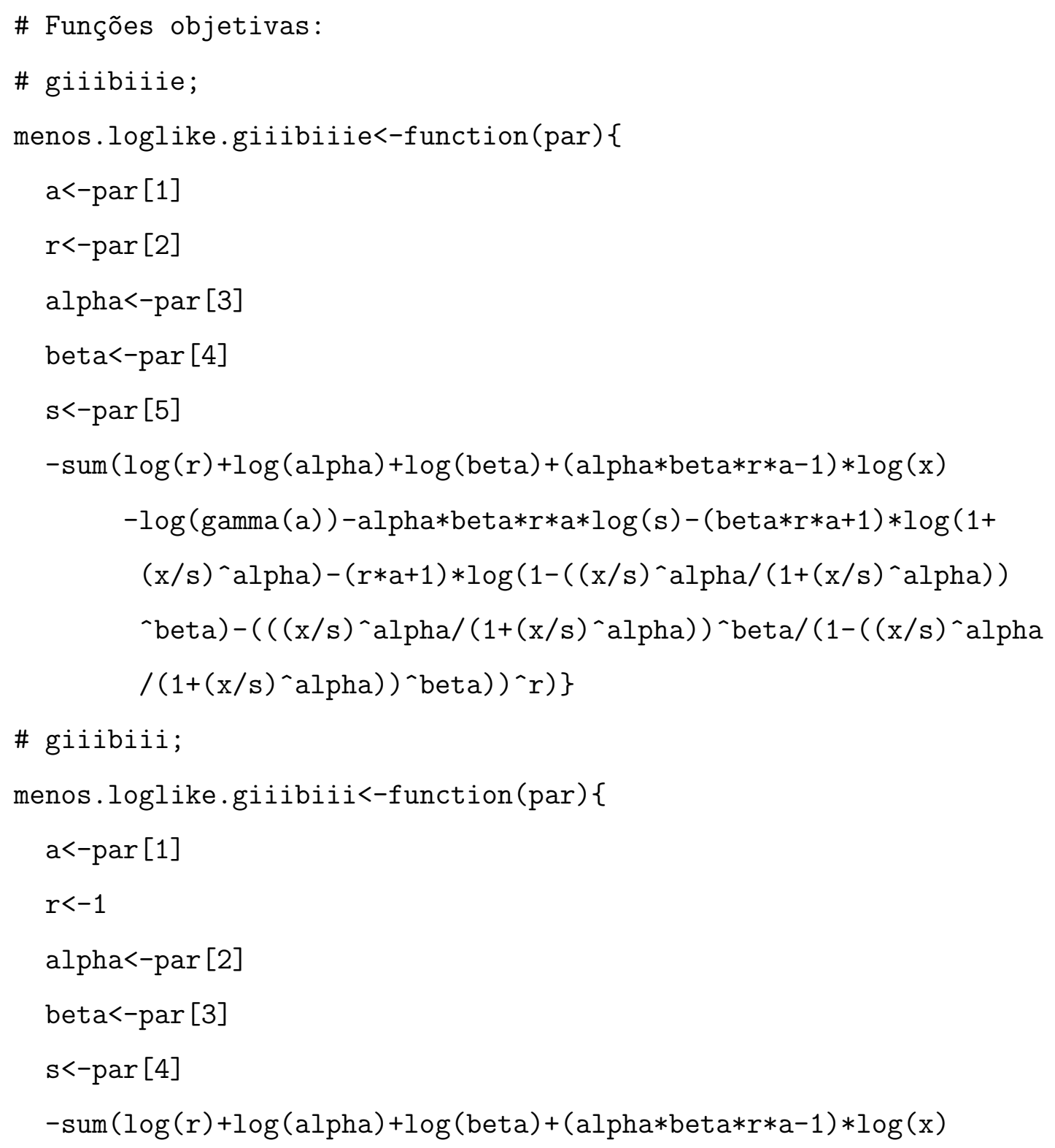




$$
\begin{aligned}
& \text { - } \log (\text { gamma (a) ) -alpha*beta*r*a*log }(\mathrm{s})-(\text { beta*r*a+1) } * \log (1+ \\
& (\mathrm{x} / \mathrm{s}) \wedge a l p h a)-(\mathrm{r} * \mathrm{a}+1) * \log (1-((\mathrm{x} / \mathrm{s}) \wedge \mathrm{alpha} /(1+(\mathrm{x} / \mathrm{s}) \wedge a l p h a)) \\
& \text { `beta })-\left(((x / s) \wedge a l p h a /(1+(x / s) \wedge a l p h a))^{\wedge} \operatorname{beta} /(1-((x / s)\right. \\
& \text { `alpha/(1+(x/s)^alpha } \left.\left.\left.\left.))^{\wedge} \text { beta }\right)\right)^{\wedge} r\right)\right\}
\end{aligned}
$$

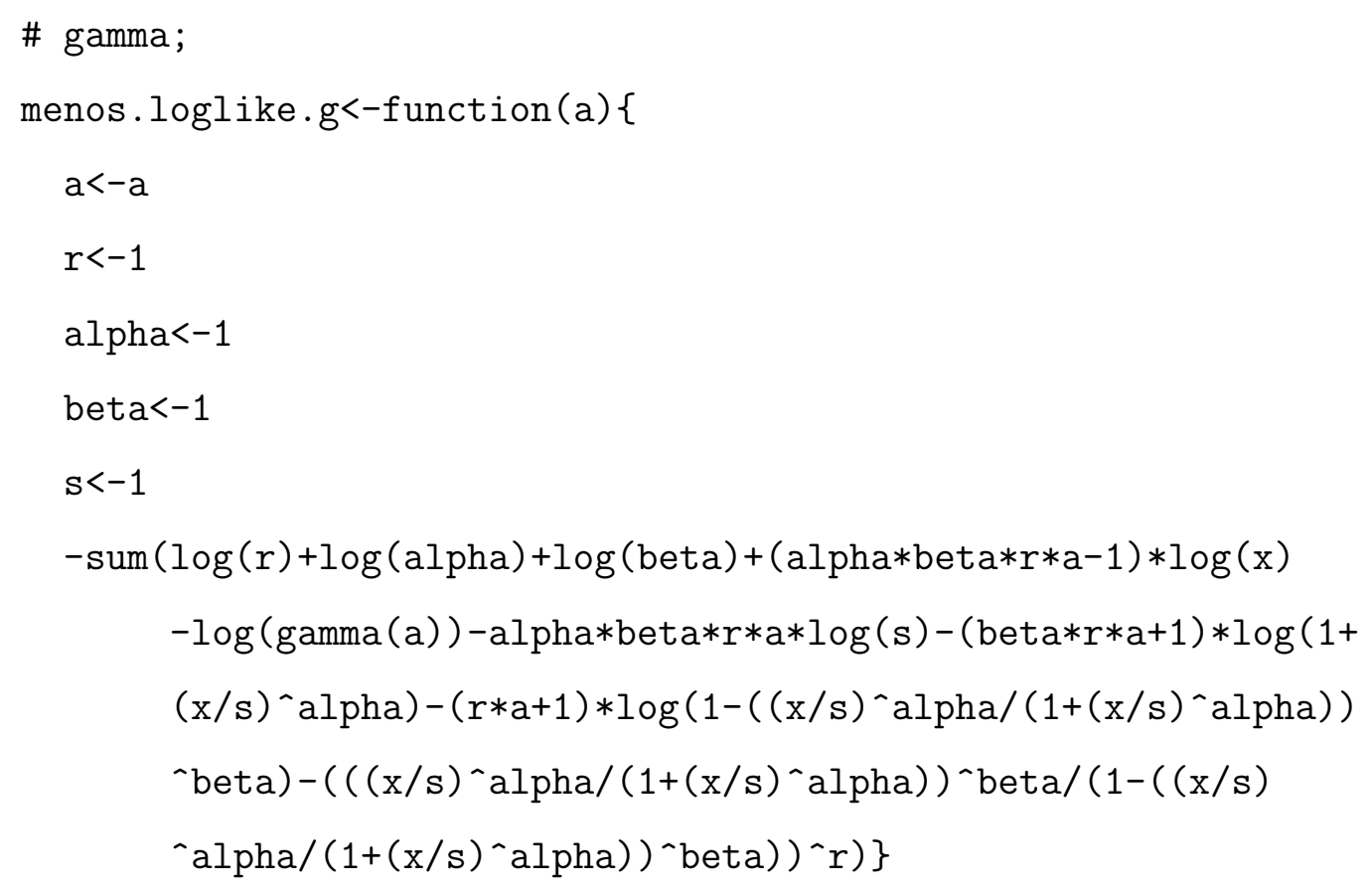


\# giibiiig vs g (hypotheses $\mathrm{HO}: \mathrm{r}=\mathrm{al}$ pha=beta=s=1);

w1<-2*(results.g\$value-results.giiibiiie\$value)

w1

pvalue $1<-1-$ pchisq (w1, df=4)

pvalue1

\# Gráfico

hist $(x, \operatorname{prob}=\mathrm{T}, \mathrm{ylim}=\mathrm{c}(0,0.7)$, main $="$ ",ylab=" $\mathrm{f}(\mathrm{x}) ")$

$\operatorname{dom}<-\operatorname{seq}(0.01,150,0.01)$

\# giiibiiig

a=results.giiibiiie\$par[1]

r=results .giiibiiie\$par [2]

alpha=results.giibiiie\$par [3]

beta=results .giiibiiie\$par [4]

$\mathrm{s}=$ results . giiibiiie\$par [5]

ima $<-f d p$ (dom)

lines (dom, ima, type="l")

\# giiibiii

a=results .giiibiii\$par [1]

$r=1$

alpha=results $\cdot$ giiibiii\$par [2]

beta=results .giiibiii\$par [3]

$\mathrm{s}=$ results . giiibiii\$par [4]

ima $<-f d p$ (dom)

lines (dom, ima, lty=2)

\# $\operatorname{gamma}(\mathrm{a}, 1)$

$\mathrm{a}=$ results. $\mathrm{g} \$ \mathrm{par}[1]$

$r=1$

alpha $=1$

beta $=1$

$\mathrm{s}=1$

ima $<-f d p$ (dom) 
lines (dom, ima, 1 ty $=3$ )

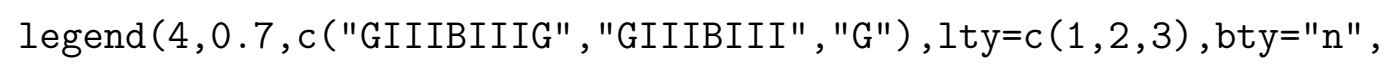

\#\#\# Programação da GIIIWG \#\#\#

\# fdp da GWIIIE:

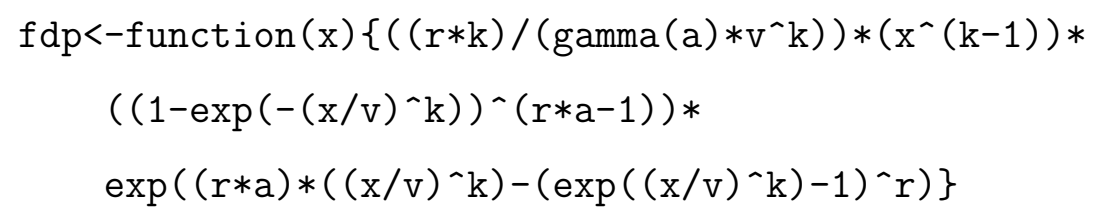




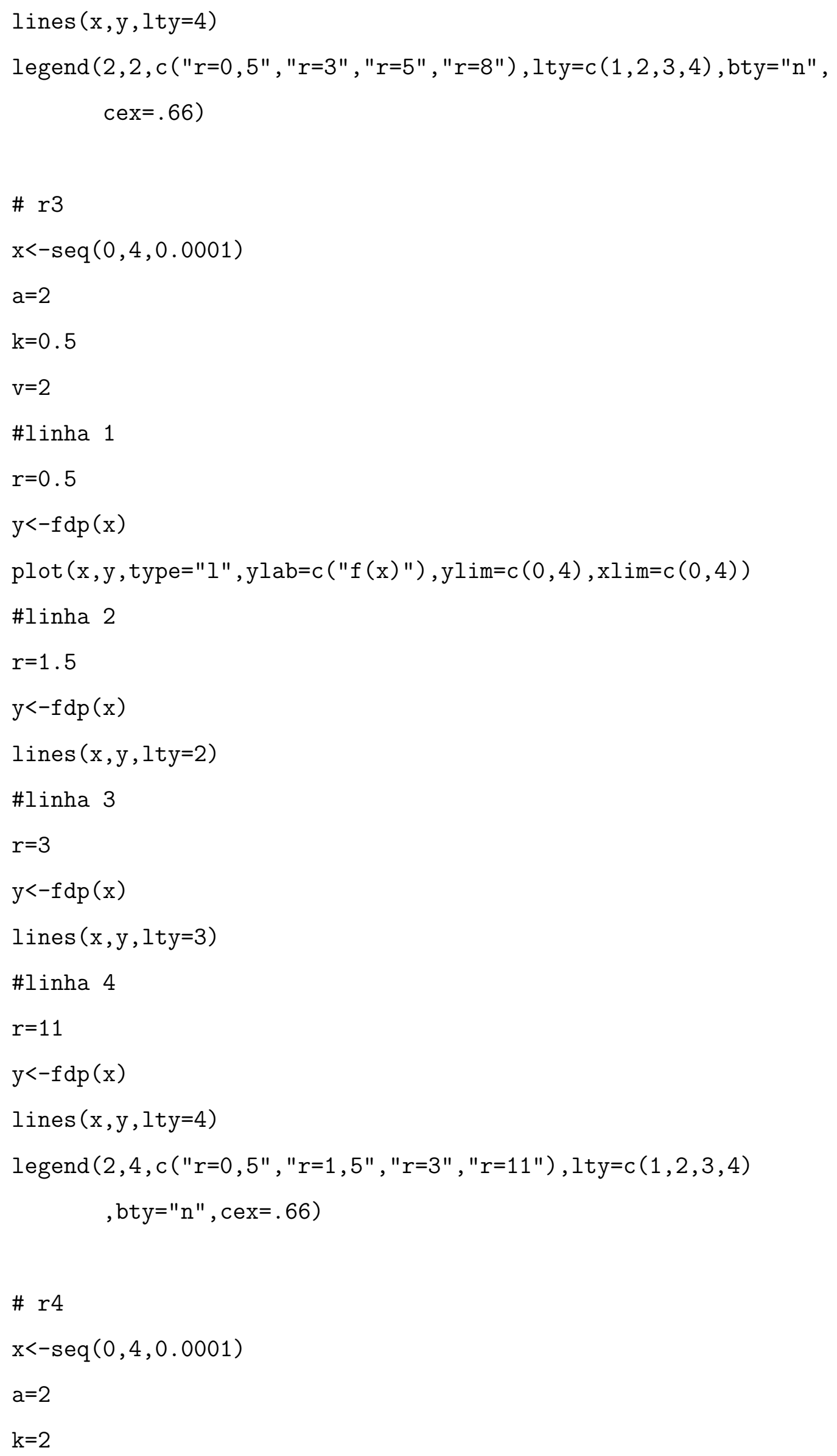


$\mathrm{v}=2$

\#linha 1

$r=0.1$

$\mathrm{y}<-\mathrm{fdp}(\mathrm{x})$

plot $(x, y$, type $=" 1 ", y l a b=c(" f(x) "), y \lim =c(0,5), x \lim =c(0,4))$

\#linha 2

$r=1.5$

$y<-f d p(x)$

$\operatorname{lines}(\mathrm{x}, \mathrm{y}, \mathrm{lty}=2)$

\#linha 3

$r=3$

$\mathrm{y}<-\mathrm{fdp}(\mathrm{x})$

lines $(x, y, 1$ ty $=3)$

\#linha 4

$r=5.5$

$\mathrm{y}<-\mathrm{fdp}(\mathrm{x})$

lines $(x, y, l$ ty $=4)$

legend $(2.5,5, c(" r=0,1$ " , "r=1, 5" , "r=3", "r=5, 5"), lty=c $(1,2,3,4)$

, bty $=" n ", c e x=.66$ )

\# Gráf. da txf da GIIIWG:

\# acumulada da GIIIGW:

fda<-function (x)\{pgamma (

\# taxa de falha:

$\mathrm{h}<-$ function $(\mathrm{x})\{\mathrm{fdp}(\mathrm{x}) /(1-\mathrm{fda}(\mathrm{x}))\}$

\# txf a1

$r=1.5$

$\mathrm{k}=1.1$

$\mathrm{v}=1.3$

$x<-\operatorname{seq}(0.0001,2,0.0001)$

\#linha 1 
$\mathrm{a}=0.01$

$\mathrm{y}<-\mathrm{h}(\mathrm{x})$

plot $(x, y$, type $=" 1 ", y l a b=c(" h(x) "), y \lim =c(0,10), x \lim =c(0,2))$

\#linha 2

$\mathrm{a}=.2$

$\mathrm{y}<-\mathrm{h}(\mathrm{x})$

$\operatorname{lines}(\mathrm{x}, \mathrm{y}, \mathrm{lty}=2)$

\#linha 3

$\mathrm{a}=.4$

$\mathrm{y}<-\mathrm{h}(\mathrm{x})$

lines $(x, y, l$ ty $=3)$

\#linha 4

$a=.5$

$\mathrm{y}<-\mathrm{h}(\mathrm{x})$

lines $(x, y, l$ ty $=4)$

legend $(1.5,6, c(" a=0,01 "$, " $a=0,2 ", " a=0,4 ", ~ " a=0,5 "), 1$ ty=c $(1,2,3,4,5)$, bty $=" n ", \operatorname{cex}=0.66$ )

$\#$ txf a2

$r=2.5$

$\mathrm{k}=0.5$

$\mathrm{v}=3$

\#abcissa

$x<-\operatorname{seq}(0.0001,8,0.0001)$

\#linha 1

$a=1$

$\mathrm{y}<-\mathrm{h}(\mathrm{x})$

plot $(x, y$, type $=" 1 ", y l a b=c(" h(x) "), x \lim =c(0,7), y \lim =c(0,10))$

\#linha 2

$\mathrm{a}=2$

$\mathrm{y}<-\mathrm{h}(\mathrm{x})$

lines $(x, y, l t y=2)$ 
\#linha 3

$a=3$

$\mathrm{y}<-\mathrm{h}(\mathrm{x})$

$\operatorname{lines}(\mathrm{x}, \mathrm{y}, \mathrm{lty}=3)$

\#linha 4

$\mathrm{a}=4$

$\mathrm{y}<-\mathrm{h}(\mathrm{x})$

$\operatorname{lines}(\mathrm{x}, \mathrm{y}, \mathrm{lty}=4)$

\#linha 5

$\mathrm{a}=5$

$\mathrm{y}<-\mathrm{h}(\mathrm{x})$

lines $(x, y, 1$ ty $=5)$

legend $(0,10, c$ ("a=1" , "a=2", "a=3" , "a=4" , "a=5"), 1ty=c $(1,2,3,4,5)$

, bty $=" n ", c e x=.66$ )

\# txf $\mathrm{r} 1$

$\mathrm{a}=0.5$

$\mathrm{k}=2.5$

$\mathrm{v}=0.5$

\#abcissa

$\mathrm{x}<-\operatorname{seq}(0.0001,1.5,0.0001)$

\#linha 1

$r=0.1$

$\mathrm{y}<-\mathrm{h}(\mathrm{x})$

plot $(x, y$, type $=" l ", y l a b=c(" h(x) "), x \lim =c(0,0.8), y \lim =c(0,30))$

\#linha 2

$\mathrm{r}=0.2$

$\mathrm{y}<-\mathrm{h}(\mathrm{x})$

lines $(x, y, 1$ ty $=2)$

\#linha 3

$r=0.3$

$\mathrm{y}<-\mathrm{h}(\mathrm{x})$ 
$\operatorname{lines}(\mathrm{x}, \mathrm{y}, \mathrm{lty}=3)$

\#linha 4

$r=0.5$

$\mathrm{y}<-\mathrm{h}(\mathrm{x})$

$\operatorname{lines}(\mathrm{x}, \mathrm{y}, \mathrm{lty}=4)$

\#linha 5

$r=0.8$

$\mathrm{y}<-\mathrm{h}(\mathrm{x})$

$\operatorname{lines}(\mathrm{x}, \mathrm{y}, 1 \mathrm{ty}=5)$

legend (0.05, 30, c ("r=0 , 1" , "r=0.2" , "r=3" , "r=0 , 5" , "r=0, 8")

, lty $=c(1,2,3,4,5)$, bty $=" n "$, cex $=0.65)$

\# txf r2

$a=2.5$

$\mathrm{k}=0.5$

$\mathrm{v}=1.7$

\#abcissa

$\mathrm{x}<-\operatorname{seq}(0.001,10,0.001)$

\#linha 1

$r=0.5$

$\mathrm{y}<-\mathrm{h}(\mathrm{x})$

plot $(x, y$, type $=" 1 ", y l a b=c(" h(x) "), y \lim =c(0.1,0.5), x \lim =c(0,4))$

\#linha 2

$r=0.6$

$\mathrm{y}<-\mathrm{h}(\mathrm{x})$

$\operatorname{lines}(\mathrm{x}, \mathrm{y}, 1 \mathrm{ty}=2)$

\#linha 3

$r=0.7$

$\mathrm{y}<-\mathrm{h}(\mathrm{x})$

$\operatorname{lines}(\mathrm{x}, \mathrm{y}, \mathrm{lty}=3)$

\#linha 4

$r=0.8$ 


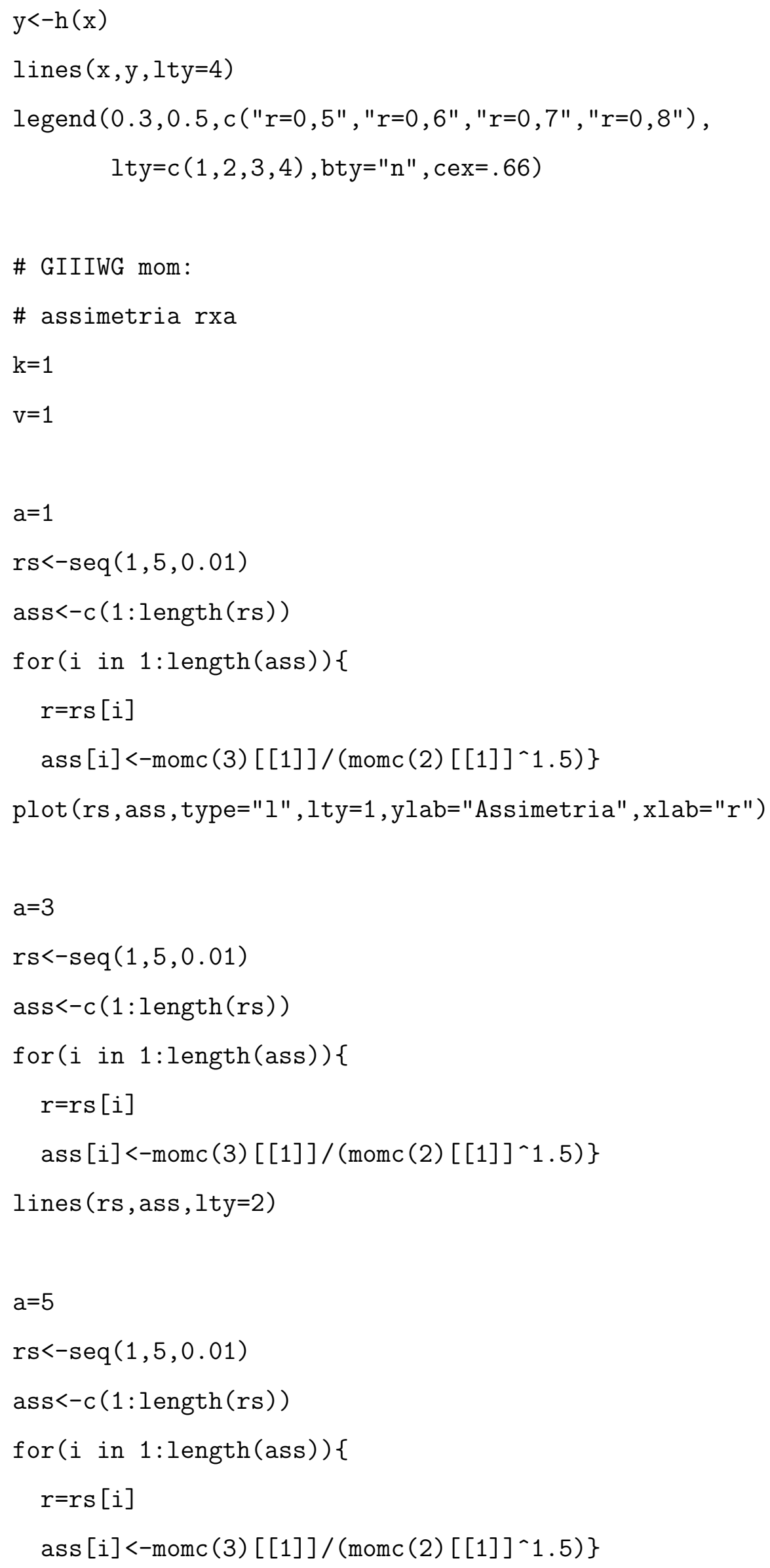




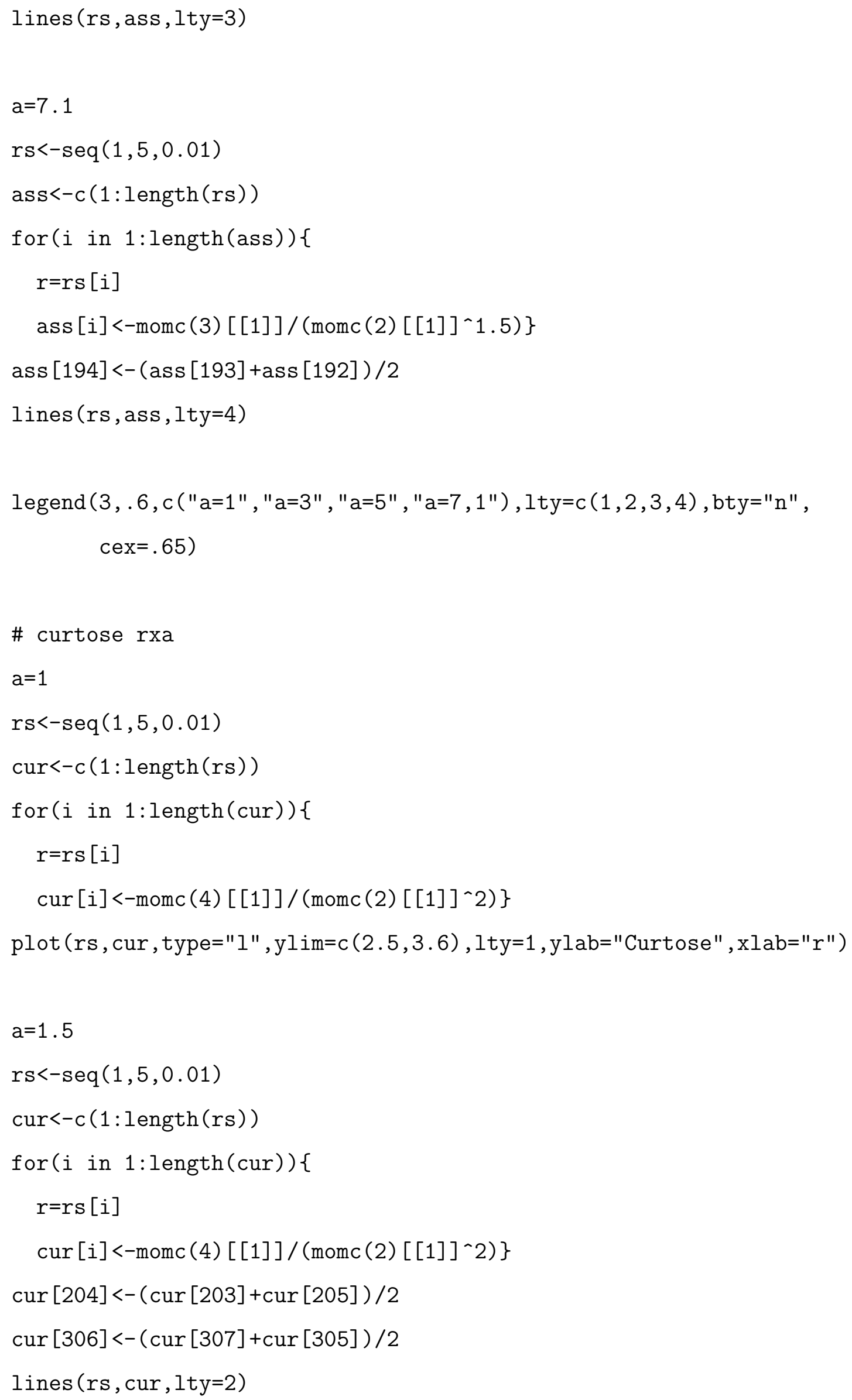




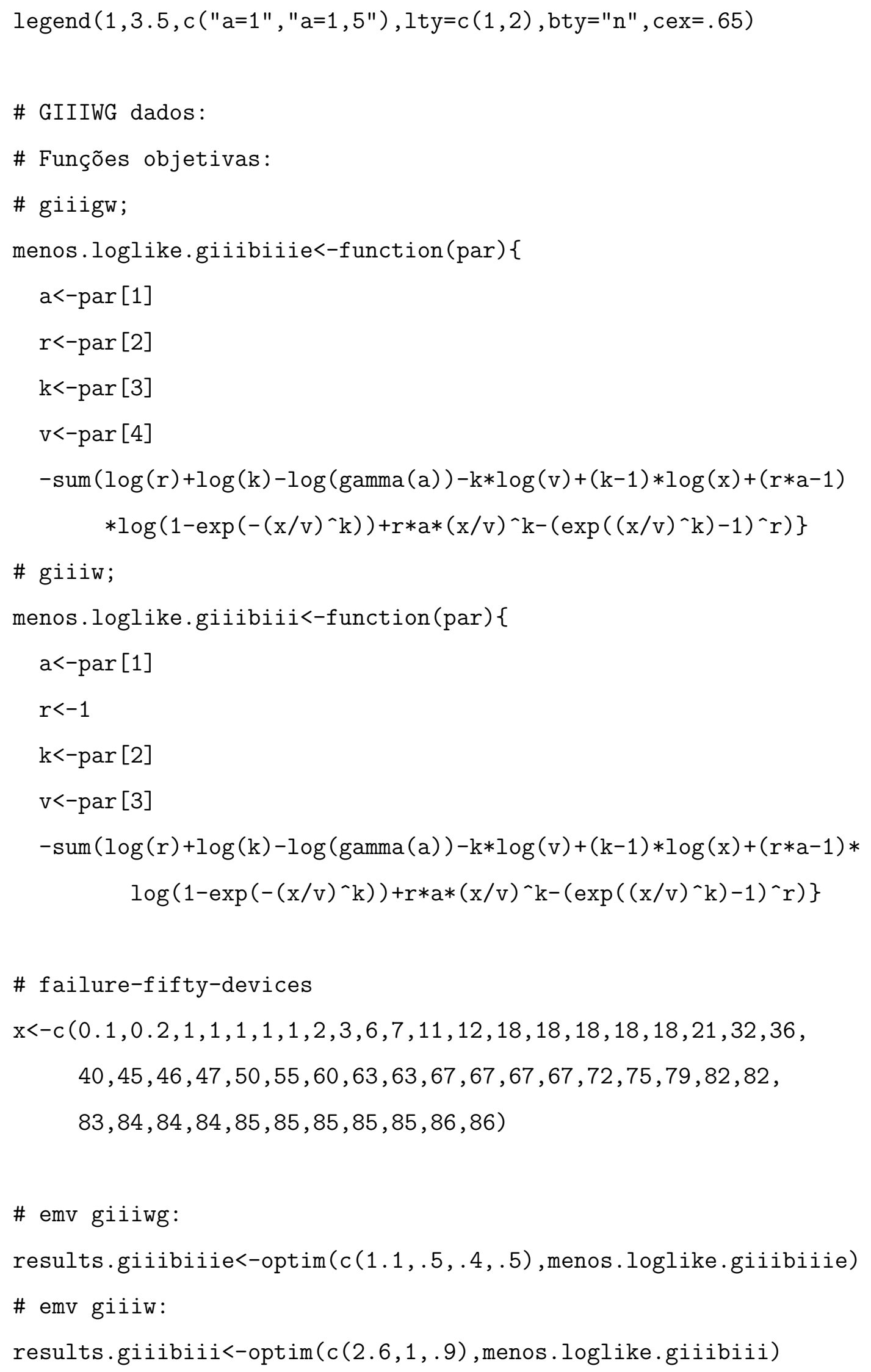


\# Teste da Razão de Verossimilhança Generalizada:

\# giiiwg vs giiiw (hypotheses HO:r=1);

w<-2*(results.giibiii\$value-results.giibiiie\$value)

W

pvalue $<-1$-pchisq $(w, d f=1)$

pvalue

\# Gráfico

hist $(x$, prob=T, main=" ", $y l a b=" f(x) ")$

dom<-seq $(0.01,150,0.01)$

\# giiibiiig

$\mathrm{a}=$ results .giibiiie\$par [1]

r=results.giibiiie\$par [2]

k=results.giibiiie\$par [3]

v=results .giiibiiie\$par [4]

ima $<-f d p(d o m)$

lines (dom, ima, type="l")

\# giibiii

a=results.giibiii\$par [1]

$r=1$

k=results.giiibiii\$par [2]

v=results.giiibiii\$par [3]

ima $<-f d p(d o m)$

lines (dom, ima, lty $=2$ )

legend (25, 0.025, c ("GIIIWG" , "GIIIW") , lty=c $(1,2)$, bty="n" , cex=0.75) 